\title{
Functional Reorganization in Somatosensory Cortical Areas 3b and 1 of Adult Monkeys After Median Nerve Repair: Possible Relationships to Sensory Recovery in Humans
}

\author{
John T. Wall, Jon H. Kaas, Mriganka Sur, ${ }^{1}$ Randall J. Nelson, ${ }^{2}$ Daniel J. Felleman, ${ }^{3}$ and \\ Michael M. Merzenich ${ }^{4}$ \\ Department of Psychology, Vanderbilt University, Nashville, Tennessee 37240
}

Previous studies have shown that the primary somatosensory cortex of adult mammals undergoes somatotopic reorganization in response to peripheral nerve transection. The present study assesses how cortical organization is affected when a transected nerve subsequently regenerates. The median nerve to one hand of adult owl monkeys was transected and repaired. Following nerve regeneration, the representations of the hand in cortical areas $3 \mathrm{~b}$ and 1 were studied with neurophysiological mapping methods.

The major results were as follows: (1) Peripherally, median nerve transection, repair, and regeneration resulted in reinnervation of the median nerve skin territory. (2) Centrally, both the initial loss and subsequent regeneration of median nerve inputs caused reorganizational changes in cortex. (3) Reorganizational changes were specifically restricted to regions of the hand cortex where inputs from the median nerve were normally represented. (4) The functional features of cortical regions that recovered tactile responsiveness from reinnervated skin regions were abnormal in several respects. Most notably, these regions (a) contained recording sites with abnormally located or multiple cutaneous receptive fields, and (b) contained major topographical changes, such as reestablishment of palmar pad or digit representations in small, discontinuous patches of cortex. (5) Normal organizational features were reestablished to a more limited extent. These features included recovery of delimited, discrete receptive fields and reestablishment of topographic representations for localized skin areas. (6) Different transformations in topographical organization were seen in areas $3 \mathrm{~b}$ and 1 of the same monkey.

These results suggest that nerve regeneration reestablishes the cortical capacity to process tactile information from reinnervated skin via a prolonged reorganizational process that appears dependent on peripheral and central factors. Cortical recovery mechanisms clearly appear to have limitations, since preinjury patterns of cortical organization are not widely re-

Received Mar. 11, 1985; revised July 8, 1985; accepted July 30, 1985.

We thank Drs. C. Cusick, M. Huerta, M. Sesma, and R. Wiley for their thoughtful criticisms of the manuscript. We also thank Dr. James A. McKanna for the use of his Bioquant II Image Analysis System. This work was supported by Grants NS-16446, NS10414, and BNS-8205745.

Correspondence should be sent to Dr. John T. Wall, Department of Psychology, Vanderbilt University, 134 Wesley Hall, Nashville, TN 37240.

' Present address: Section of Neuroanatomy, Yale University, New Haven, CT 06510.

${ }^{2}$ Present address: Department of Anatomy, University of Tennessee, Memphis, TN 38163.

${ }^{3}$ Present address: Department of Biology 216-76, California Institute of Technology, Pasadena, CA 91125.

4 M. M. Merzenich was a visiting scientist from Coleman Laboratory, Departments of Otolaryngology and Physiology, University of California, San Francisco, CA 94143.

Copyright @ 1986 Society for Neuroscience $0270-6474 / 86 / 010218-16 \$ 02.00 / 0$ covered even almost 1 year after repair. We suggest possible relationships between cortical reorganizational changes in these primates, and postrepair sensory changes in humans.

Recent studies in adult monkeys show that somatosensory cortical areas $3 b$ and 1 undergo significant topographical reorganization after transection injury of the median nerve to the hand (Merzenich et al., 1983a, b). Neurons in cortical zones that are deprived of median nerve inputs do not lose all cutaneous responsiveness, as one might expect, but instead become activated by "substitute" tactile inputs from adjacent hand locations that remain innervated by other nerves. The major topographical consequences of these changes are, first, loss of cortical representations of the denervated skin territory and second, a corresponding enlargement in the representations of normally innervated skin near the denervated territory (Fig. 1). Considered together with similar findings from denervation studies in other mammals (for recent review see Kaas et al., 1983), these studies demonstrate that the central somatosensory system retains a lifelong ability to reorganize in response to changes in the integrity of peripheral inputs.

The injury procedures used in denervation studies cause a sustained loss of low threshold mechanoreceptor inputs from the denervated skin. Central reorganization under these conditions reflects a "readjustment" to injury of inputs, rather than an actual "recovery" of function in the sense of a restoration of central activation from the denervated skin. Recovery, in this sense, requires regeneration of injured inputs and reinnervation of the skin. The present experiments assessed regeneration-related central recovery by studying topographical organization in the hand representations of cortical areas $3 \mathrm{~b}$ and 1 of adult monkeys that had undergone transection, repair, and regeneration of the median nerve. Three major issues were of interest. First, are cortical reorganizational changes resulting from nerve transection injury reversible if the injured nerve subsequently regenerates? This issue is important for a general understanding of the sequence of events underlying cortical recovery after injury of ncrves. Sccond, is normal cortical organization reestablished after nerve repair and regeneration? This issue is important for understanding central recovery capacities after nerve repair. Third, what functional abnormalities result after nerve repair? This question is directly relevant for recognizing limitations in central recovery mechanisms after nerve regeneration.

\section{Materials and Methods}

Four adult owl monkeys (Aotus trivirgatus) which had undergone transection and repair of the median nerve to one hand were studied 76$322 \mathrm{~d}$ after repair. Detailed maps of the complete hand representation were made in cortical area $3 b$ of three monkeys and in area 1 of two monkeys. In monkeys whose areas $3 b$ or 1 were not completely studied, 


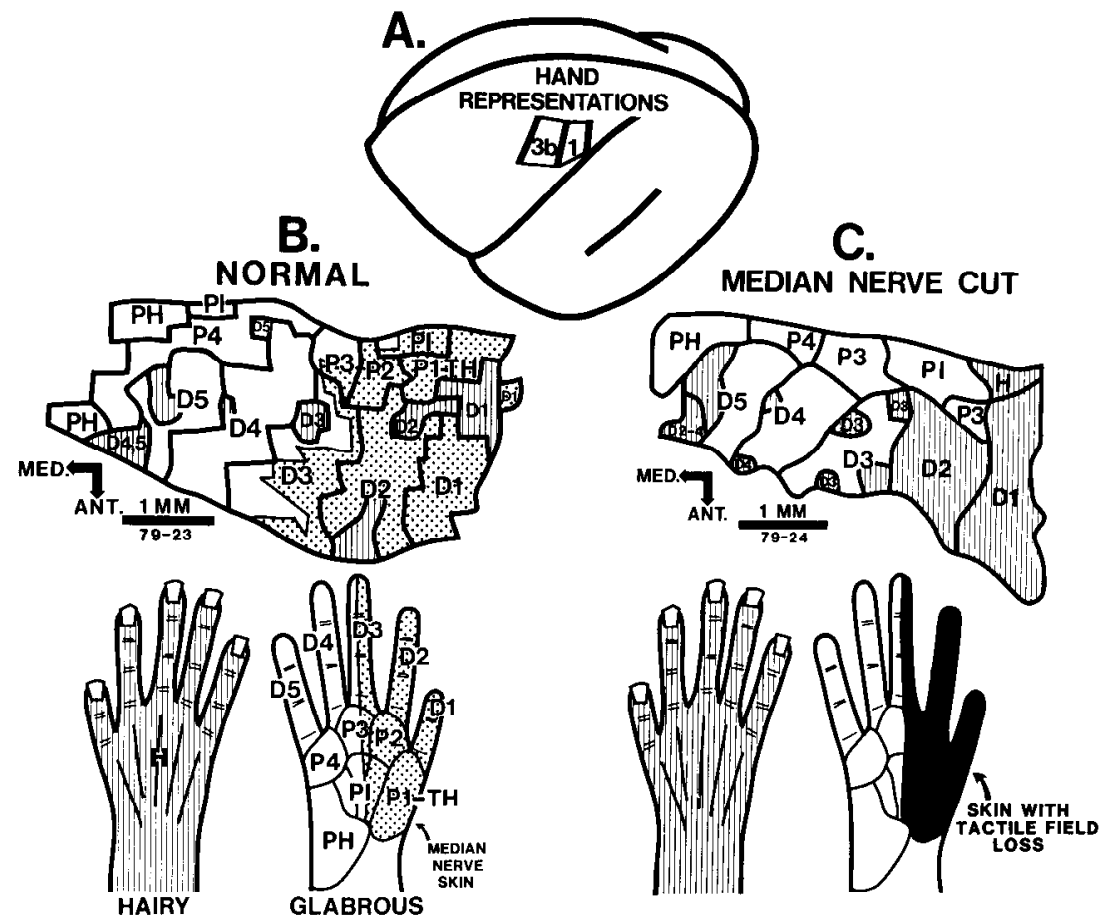

Figure 1. Somatosensory cortical representations of the hand in owl monkeys with normal and transected median nerves to the hand. $A$, Area $3 \mathrm{~b}$ and 1 hand representations are located in a flat region on the dorsolateral surface of parietal cortex. $B$, Hand representation in area $3 \mathrm{~b}$ of a normal owl monkey. Cutaneous inputs from the glabrous (unhatched and stippled) and hairy (hatched) hand surfaces are topographically represented in area $3 \mathrm{~b}$. The median nerve innervates the thumbward half of the glabrous hand (bottom, stippled), and normally provides inputs to the lateral part of the hand representation (top, stippled). PH, Hypothenar pad; $P I$, insular pads; $P 1-T H$, pad 1 and thenar pad; $P 2-4$, pads 2-4; D1-5, digits $1-5 ; H$, hairy hand proximal to knuckles. $C$, Hand representation in area $3 \mathrm{~b}$ of an owl monkey with a transected and chronically ligated median nerve (from Merzenich et al., 1983a). No area $3 \mathrm{~b}$ neurons had cutaneous receptive fields on skin regions normally innervated by the median nerve (bottom, blackened zone on hand), but, due to the nerve injury, the lateral part of the hand representation (top) had reorganized and acquired substitute inputs from hairy (hatched) and glabrous (unhatched) skin regions bordering the denervated skin (e.g., hairy D1 and 2, glabrous P3 and I). Arca $3 \mathrm{~b}$ hand represcntations illustratcd in $B$ and $C$ have been rotated counterclockwise $90^{\circ}$ from the orientation shown in $A$.

high-density maps were made of more limited portions of the hand representation. All illustrated results and quantitative measures were taken from monkeys with fully defined hand maps. Complete maps of the hand representation in area $3 \mathrm{~b}$ and complete or partial maps in area 1 were made in three additional owl monkeys with normal nerves, to provide control data for quantitative comparisons (e.g., Fig. 1B). Cortical maps defined in other studies of normal monkeys (Merzenich et al., 1978; Wall et al., 1983) or in studies of monkeys with transected and ligated median nerves (Merzenich et al., 1983a, b; e.g., Fig. 1C) were also referred to for comparative purposes.

Nerve transection and repair procedures. Monkeys were anesthetized with ketamine hydrochloride (25-50 $\mathrm{mg} / \mathrm{kg}$. IM; White et al., 1982) and the skin on one forearm was locally anesthetized with $5 \%$ xylocaine. Aseptic procedures were used to isolate several millimeters of the median nerve at a level just proximal to the wrist. The nerve was transected with a scalpel and the cut ends were immediately reconnected with epineural sutures (10-0) under microscopic view. The incision was closed and prophylactic antibiotics were given for the next week. All monkeys recovered without complication.

Mapping procedures. The neurophysiological recording procedures were similar to those used in previous studies (Merzenich et al., 1978, 1983a, b; Wall et al., 1983). During surgery and mapping, animals were anesthetized with ketamine hydrochloride $(25-50 \mathrm{mg} / \mathrm{kg}$, IM) and body temperature was maintained at $37^{\circ} \mathrm{C}$ with a water pad. The scalp was opened, a craniotomy was made over the anterior parietal cortex, and a chamber was constructed around the craniotomy with dental acrylic. The dura was reflected toward the margins of the craniotomy, the chamber filled with silicone, and a magnified photograph (25-30x) made of the exposed cortical surface.

Parylene-insulated tungsten microelectrodes (1-2 $\mathrm{M} \Omega$ at $1 \mathrm{kHz})$ were directed toward the cortex at an angle normal to the surface. Under microscopic view, the penetration location was marked on the photograph and the electrode was advanced into the cortex, while the hand was tactilely explored with fine-tipped probes. Several hundred penetrations, separated by about $200-300 \mu \mathrm{m}$, were systematically spaced across areas $3 b$ and 1 . In each penetration, specific attempts were made to define cutaneous receptive fields from multiple-unit discharges evoked by mechanical stimuli that produced a just-visible indentation of the

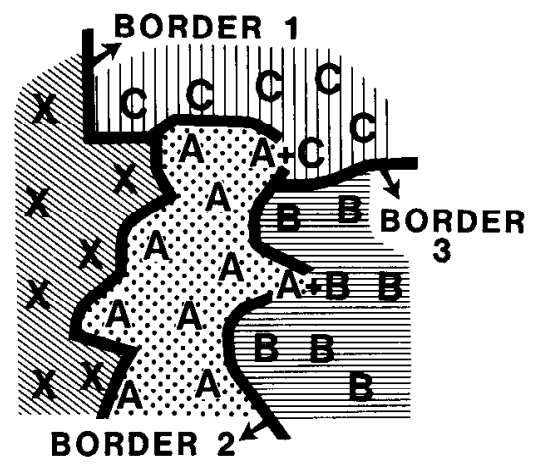

Figure 2. Methods used to delineate cortical areas with different inputs. Each cortical penetration was first identified by the response properties of neurons at that site (e.g., $A, B, C$ s $=$ sites with receptive fields on different glabrous skin regions; $X$ 's $=$ sites with fields on a hairy skin region). Borders were established midway between adjacent penetrations that had different properties (e.g., Border 1 ). If a single cutaneous field straddled two skin regions that were being distinguished-for example, penetrations $A+B$ and $A+C$-the border separating the cortical areas was placed at the penetration location (e.g., Borders 2 and 3). These rules were also generalized to show the distribution of cortical areas with other input differences. 

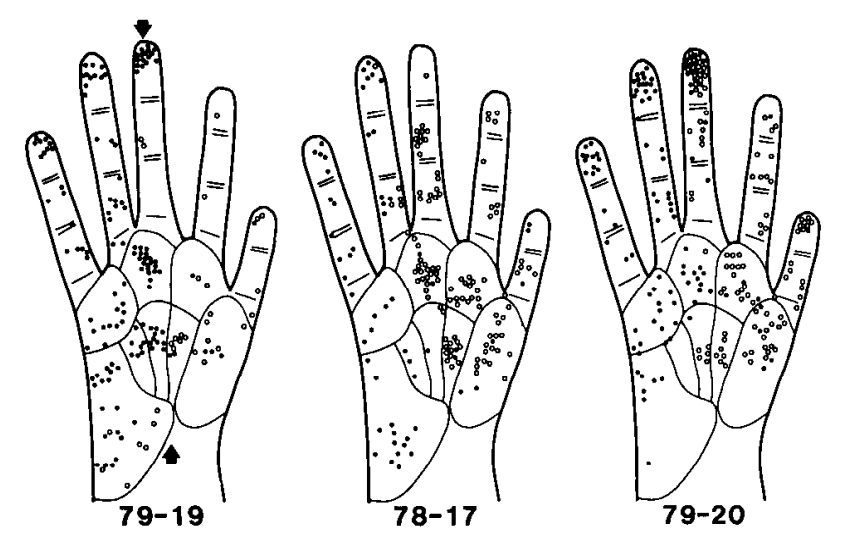

Figure 3. Centers of all glabrous skin fields recorded in complete maps of the area $3 \mathrm{~b}$ hand representations of three monkeys with repaired nerves. Postrepair times were 76 (left), 153 (middle), and 322 (right) d. The cortical sampling density was similar in all cases. Note: The median nerve territory (e.g., skin to the right of the arrows in 79-19) was reinnervated in all cases; centers associated with cortical sites having a single field (๑) are distinguished from centers associated with sites with multiple fields $(O)$ to show that abnormal multiple field centers were in or near the median nerve innervation territory; the density of reinnervated fields increased with longer postrepair times, especially at more distal skin locations.

skin. When responses to cutaneous stimuli were apparent, receptive field boundaries were carefully defined and drawn on detailed illustrations of the hand. In penetrations where responses to cutaneous stimuli were not evoked, efforts were made to determine whether there were responses to stimulation of muscles, joints, or other subcutaneous inputs. Receptive fields were usually defined in detail at depths of 600 $1100 \mu \mathrm{m}$ below the surface; however, neurons recorded at other depths in a given penetration typically had receptive fields similar to the fields defined at those depths.

During each experiment, microlesions $(10 \mu \mathrm{A}, 10 \mathrm{sec})$ were made at selected recording sites. Upon completion of the experiment, the animals were decply ancsthetized and perfused with saline and $10 \%$ formalin. The brain was removed, blocked, immersed in 30\% sucrose formalin, and frozen-sectioned in a parasagittal plane. Cresyl violetstained sections were used to define the architectonic borders of areas $3 \mathrm{~b}$ and 1 , and to relate these borders to the microlesions in the hand maps.

Data analysis. Owl monkeys do not have a central sulcus and the hand representations in areas $3 \mathrm{~b}$ and 1 are located over a flat and directly accessible region on the surface of the hemisphere (Fig. $1 A$ ). This accessibility offered a great practical advantage in the construction of detailed, two-dimensional maps of cortical activation patterns. To construct cortical maps, each penetration on the brain photograph was labeled according to how neurons at that site responded. Sites with cutaneous responses were further identified by their receptive field location. To detcrminc the cutancous representation of a delimited subregion of the hand, such as a digit or palmar pad, the relevant skin zone was defined on the hand and all cortical penetrations with a receptive field in that skin zone were identified. Borders delimiting cortical zones with a similar property were placed midway between adjacent penetrations that did or did not have that property (Fig. 2). If a single cutaneous receptive field straddled two skin regions that were being distinguished, the border between the cortical representations of the two skin regions was placed at the penetration location (Fig. 2). As is discussed in Results cortical penetrations in monkeys with repaired nerves sometimes had more than one receptive field component. By considering receptive field components on different skin regions separately, the above rules were also applied to these penetrations so that consistent comparisons could be made of the representations of normally innervated and reinnervated subregions of the hand. Using these proccdures, it was possible to determine the location and distribution of cortical areas with different inputs. Since the cortex over which the hand representations extend is relatively flat, the sizes of cortical areas could be accurately measured with a planimeter (Bioquant image analysis system, E. Leitz).
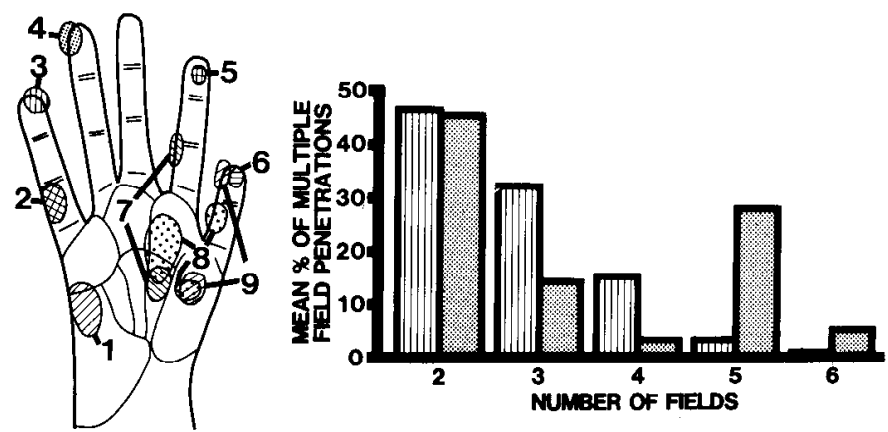

Figure 4. Cutaneous receptive fields, defined at normal and reinnervated skin. (Lefi) Examples of receptive fields from multiple-unit responses recorded at single cortical sites in area $3 \mathrm{~b}$ after nerve repair. Fields 1-4 were located on normally innervated glabrous skin and are similar to fields recorded in normal monkeys. Fields 5 and 6 are examples of reinnervated fields recorded at two SRF (single receptive field) sites, while 7-9 are examples of reinnervated fields recorded at three MRF (multiple receptive field) sites. (Right) Distribution of the number of fields seen per MRF site in areas 3b (hatched) and 1 (stippled). In area $3 \mathrm{~b}$, most MRF penetrations had 2 or 3 fields. A large proportion of MRF penetrations in area 1 had 2 or 3 fields; however, a sizable proportion also had more fields than were seen in area $3 \mathrm{~b}$.

\section{Results}

\section{Reinnervation of the median nerve skin territory}

As determined by previous nerve recording (Wall and Kaas, unpublished results) and transection and ligation (Merzenich et al., 1983a, b) studies, the median nerve provides all or nearly all of the low-threshold mechanoreceptor innervation to the glabrous skin on digits 1,2 , part of digit 3 , the first and thenar pads, the second pad, and portions of the insular and third pads (Fig. $1 B$ ). In the present study, forearm dissections indicated that the repaired median nerves crossed the repair sites and extended into their normal zone in the radial hand. Areas $3 \mathrm{~b}$ and 1 of all monkeys with repaired nerves contained neurons with tactile receptive fields on glabrous skin surfaces normally innervated by the median nerve (e.g., Fig. 3). The recovery of these fields is attributable to regeneration, since cortical inputs from these skin surfaces are not reestablished when the median nerve is transected and prevented from regenerating by chronic ligation (e.g., Fig. 1C).

\section{Functional organization in area $3 b$}

\section{Response properties of neurons}

During penetrations into parts of area $3 \mathrm{~b}$ representing hand regions with normal radial or ulnar nerve innervation, neurons were highly responsive to light tactile stimulation of single, welldelimited cutaneous fields similar to those defined in normal monkeys (e.g., Fig. 4, fields 1-4). In contrast, the responses of neurons recorded in cortical regions with regenerated median nerve inputs were more variable and were characterized by the following properties: In $65 \%(88 / 136)$ of the recording sites with regenerated inputs, neurons were responsive to light cutaneous stimulation at more than one skin location. The centers of the receptive fields defined at these multiple receptive field (MRF) sites were in or near the innervation territory of the median nerve (e.g., Fig. 3). At any given MRF site, each receptive field component was continuous internally. Different components were separated by skin zones wherc comparable, low-threshold cutaneous stimuli did not result in activation of cortical cells. 

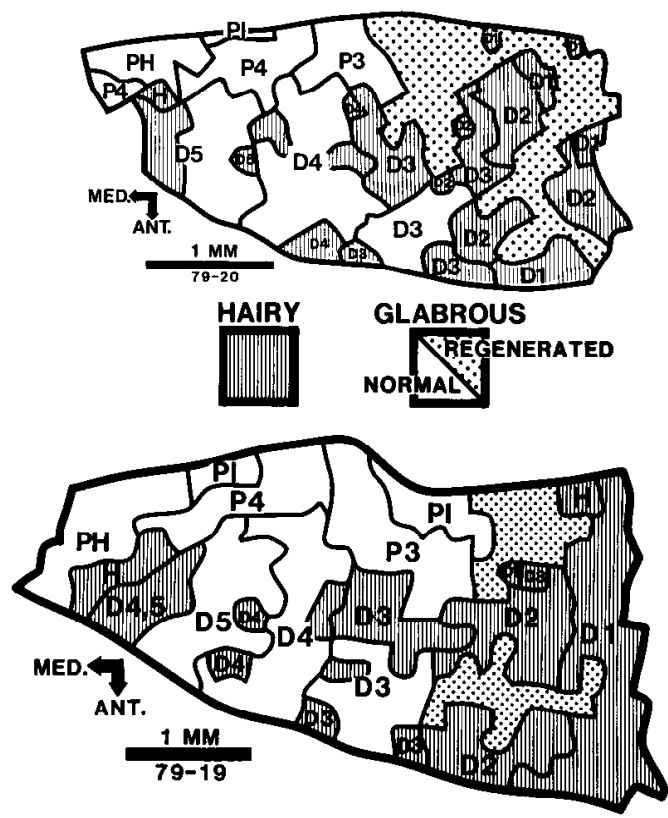

Figure 5. Hand representations in area $3 \mathrm{~b}$ of two monkeys after regeneration of the median nerve for 76 (bottom) and 322 (top) d. The representations of median nerve inputs (stippled areas) occupied a relatively normal location in the lateral part of the hand representation, but were abnormally distributed in a patch or striplike pattern. The representations of skin regions bordering the median nerve innervation territory-for example, representations of hairy digits 1-3-had expanded into cortical regions normally representing median nerve inputs. Medial parts of the hand representation appeared normal.

In addition, different components were usually distributed such that only one field was seen on any individual palmar pad or digital phalanx (e.g., Fig. 4, fields 7-9). MRF sites had up to five distinct fields, although most sites had two to three fields (Fig. 4). The combination of receptive field locations at different MRF sites varied and involved skin only on palmar pads, only on digits, or on a mixture of pads and digits. Within any single MRF penetration, the combination of fields remained relatively constant as the electrode was moved up and down a tract angled perpendicular to the cortical layers. The mean total receptive field area, derived by combining areas of all component fields per MRF site, was larger (53\%) than the mean size of a normal field, but the mean size of each individual field was smaller $(47 \%)$ than a normal field.

Neurons at $25 \%(34 / 136)$ of the cortical recording sites, with regenerated inputs, were responsive to light tactile stimulation of a single, well-defined skin location (Fig. 4, fields 5 and 6). Cutaneous fields at these single receptive field (SRF) sites were scattered across the reinnervated skin (Fig. 3) and often overlapped field components of MRF penetrations. When referenced to normal hand maps, in many instances the locations of these fields appeared inappropriate for the cortical site. The mean size of reinnervated fields at SRF sites was smaller (30\%) than the mean size of fields on normally innervated glabrous skin.

Neurons with very large receptive fields that were difficult to delimit were seen in $4 \%(6 / 136)$ of the penetrations. Neurons at these sites were exquisitely sensitive to direct tactile stimulation of the skin, to mechanical stimuli delivered indirectly by tapping the table surface on which the reinnervated hand was lying, and to high-frequency vibratory stimuli. These responses qualitatively resembled responses of afferent fibers from pacinian corpuscles (Darian-Smith, 1984) and, for this reason, we refer to these responses as "pacinian-like." Pacinian-like responses are not characteristic of neurons in the area $3 b$ hand represen-
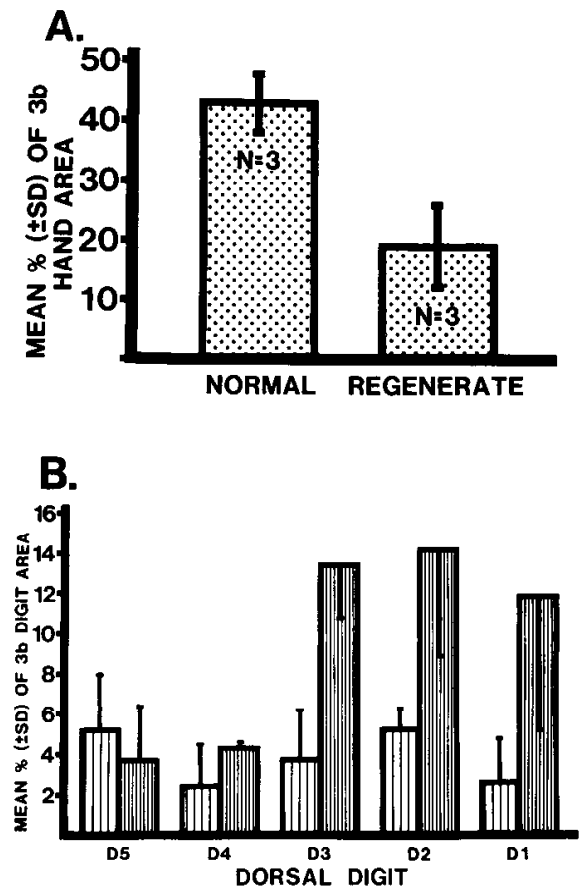

Figure 6. Areal measures of cortical representations in area $3 \mathrm{~b}$ of normal monkeys and of monkeys with repaired median nerves. $A$, The mean cortical area devoted to median nerve inputs in monkeys with repaired nerves was about half that of normal monkeys. $B$, Mean cortical areas of representations of hairy skin on digits $1-3$ in nerve repaired monkeys (narrow hatching) were three or more times larger than the representations of normal monkeys (wide hatching), whereas areas of representations of hairy skin on digits 4 and 5 were more normal.

tation of normal owl monkeys (Merzenich et al., 1978; Wall et al., 1983), or owl monkeys with chronically transected median nerves (Merzenich et al., 1983a, b). Thus, their presence in these monkeys appears to be related to nerve regeneration.

Finally, neurons in $6 \%(8 / 136)$ of the penetrations were unresponsive to light tactile stimuli and could be only weakly driven by harder taps to the radial hand. Deep receptive fields specific to joint or muscle movements were not detected, so the origin of these responses remains unclear. Weak responses to mechanical stimuli are not typical of area $3 \mathrm{~b}$ neurons in normal or chronically denervated monkeys, and thus also presumably result from regeneration-related changes.

These findings indicate that neurons in area $3 \mathrm{~b}$ were reactivated by regenerated inputs from the reinnervated skin zone. Neurons with reinnervated cutaneous fields were abnormal, however, in terms of the numbers, locations, and sizes of the skin areas to which they responded. Less frequently, normal cutaneous responses were replaced by pacinian-like responses and weak responses from abnormal cutaneous or deep inputs.

\section{Overall topography of the hand representation}

The normal topographical organization of the area $3 \mathrm{~b}$ hand representation is characterized by the following major features (e.g., Fig. 1B). Most of the hand area is devoted to the glabrous side of the hand, with receptive fields on palmar pads represented caudally and those on digits rostrally (e.g., Fig. $1 B$ ). Thus, there is an orderly proximal-to-distal progression of field locations across the hand, with caudorostral shifts in cortex. Within the representations of the palm and digit skin there is an orderly progression of inputs from the radial (thumb) to ulnar (little finger) side of the hand, with lateral-to-medial changes in cortical location (Fig. $1 B$ ). The shape and size of individual pad 

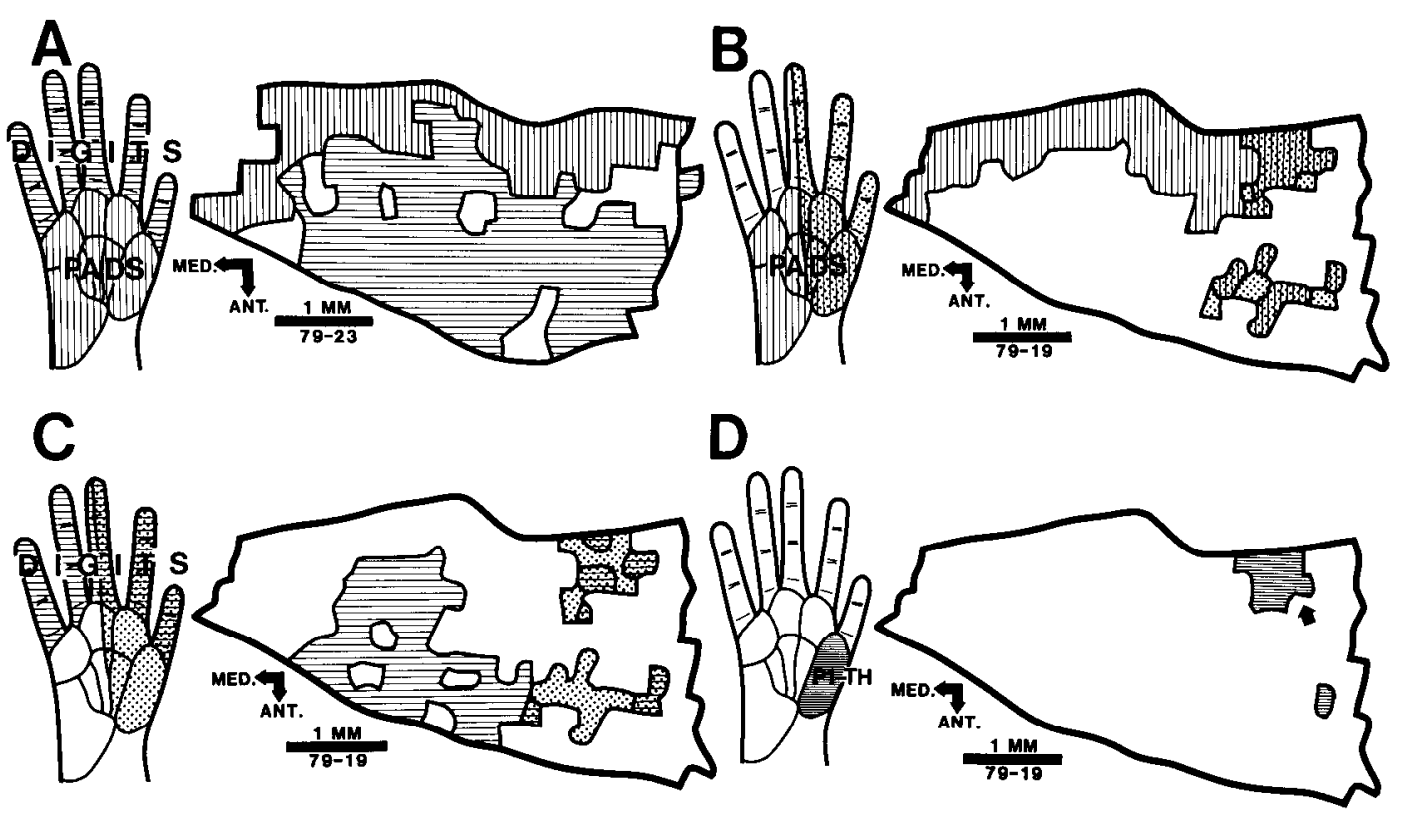

Figure 7. Topographical organization in the area $3 \mathrm{~b}$ hand representation. $A$, The topographical pattern of representation of glabrous skin on the palmar pads (vertical hatching) and digits (horizontal hatching) in a normal monkey. Note that pads are represented along the caudal margin of area $3 \mathrm{~b}$, whereas the digits are represented rostrally. $B-D$, Topographical organization in a monkey with a regenerated median nerve with $B$, the representation of the palmar pads (vertical hatching) is abnormally distributed in several patches, some of which are abnormally located in rostrolateral parts of the hand representation. Cortical regions receiving median nerve inputs are indicated by stippled areas to show that the abnormal pad representations involve only reinnervated pads. Medially in the hand representation, the pad representation is similar to normal (compare to $A$ ). $C$, Similarly, the representation of glabrous digits (horizontal hatching) is abnormally distributed in patches and, again, abnormal patches involved only reinnervated digits (compare to $A$ ). $D$. The patchlike representation of a reinnervated Pl-TH pad (horizontal hatching). Parts of this representation (arrow) appear to occupy a normal location in the hand representation (compare with P1-TH in Figure $1 B$ ).

and digit representations vary in different monkeys, but the relative location of each representation is highly predictable.

The hand representations of all monkeys with repaired nerves contained a similar general pattern of normal and abnormal topographical organization (Fig. 5). In the medial part of the hand cortex, normal topography was consistently observed in cortical areas receiving ulnar nerve inputs from skin regions on the ulnar margin of the hand (e.g., D5 and 4, P4, and PH in Figs. $1 B$ and 5 ). In contrast, lateral parts of the hand representation, which normally represent median nerve inputs, were highly abnormal in several respects. First, the total cortical area representing inputs from the regenerated median nerve was smaller than normal (stippled areas, Figs. $1 B$ and 5; see Fig. $6 A$ ). Second, the cortical zone representing median nerve inputs was located in a relatively normal position in the hand cortex, but the distribution of this representation was abnormal. For example, the representation of median nerve inputs normally extends as a continuous block across the entire rostrocaudal width of the lateral hand cortex (e.g., Fig. $1 B$ ), whereas one or more patch-or striplike areas which extended only partly across the width of the hand cortex were seen in monkeys with repaired nerves (Fig. 5). Finally, cortical representations of some hand regions that adjoin median nerve skin, but are innervated by other nerves, occupied abnormal locations in the lateral hand
Figure 8. Topographical organization in the area $3 b$ hand representation of a monkey with a repaired median nerve. All conventions as in Figure 7. $A$, Abnormal representation of the palmar pads is indicated (vertical hatching), as well as cortical regions receiving median nerve inputs (stippling). B, Abnormal representation of the glabrous digits (horizontal hatching) and cortical regions receiving median nerve inputs (stippling) are indicated. Note in $A$ and $B$ that abnormalities only occur in reinnervated skin representations. $C$ and $D$, Patchlike representations of $D 1$ and 2 , and $P 2$ : In $C$, the arrow indicates a part of the D1 representation that appears to occupy a relatively normal location in the hand area, and in $D$ the arrow indicates a correctly localized P2 patch (compare with Fig. 1B).
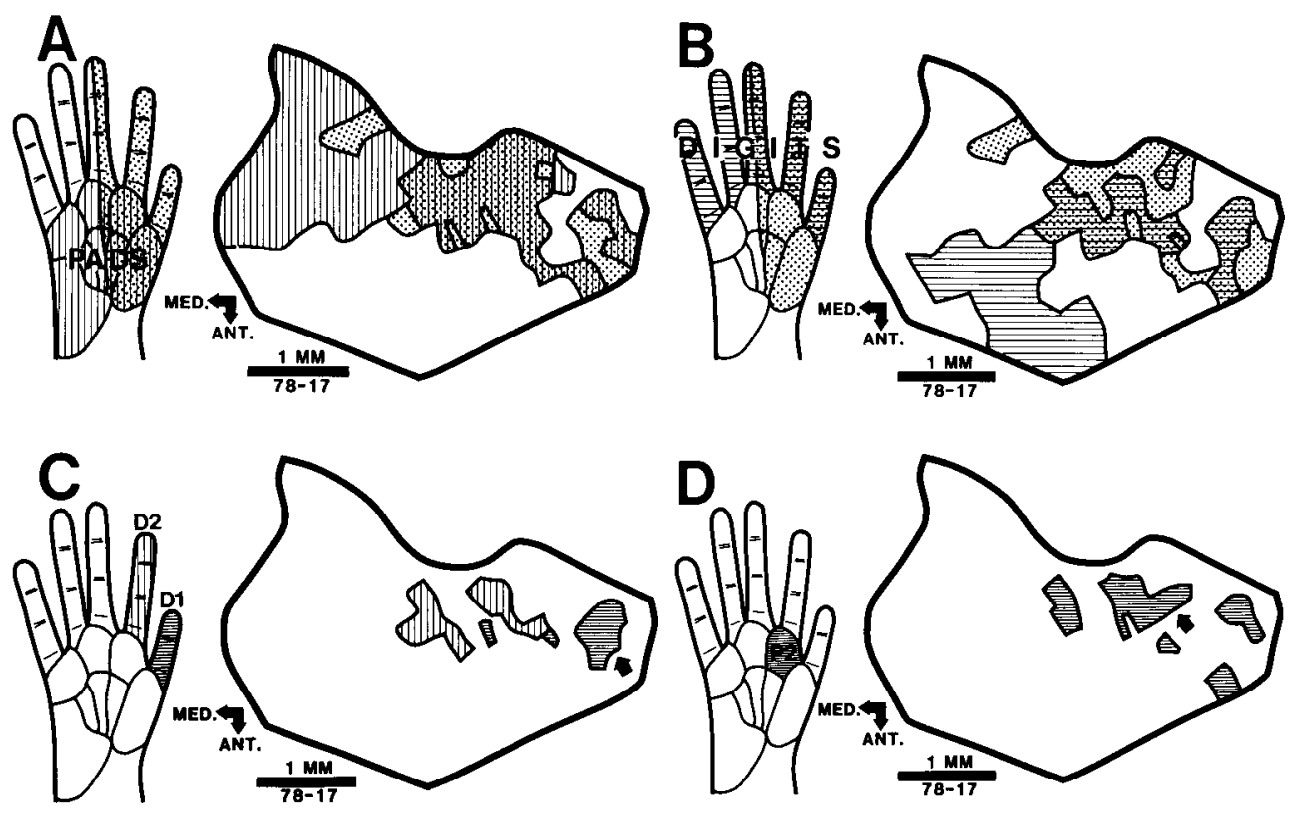

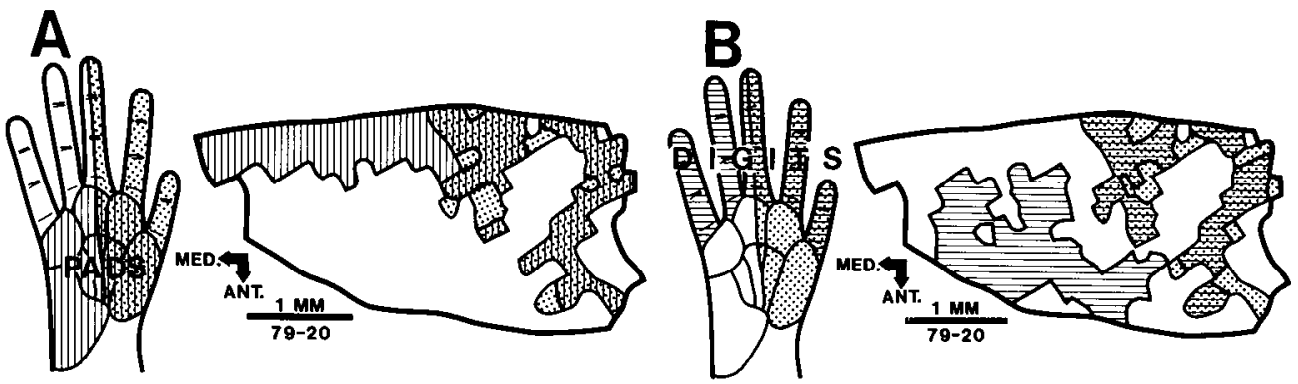

Figure 9. Topographical organization in the area $3 \mathrm{~b}$ hand representation of a monkey with a repaired median nerve. $A$ and $B$ are similar to $A$ and $B$ in Figure 8 and to $B$ and $C$ in

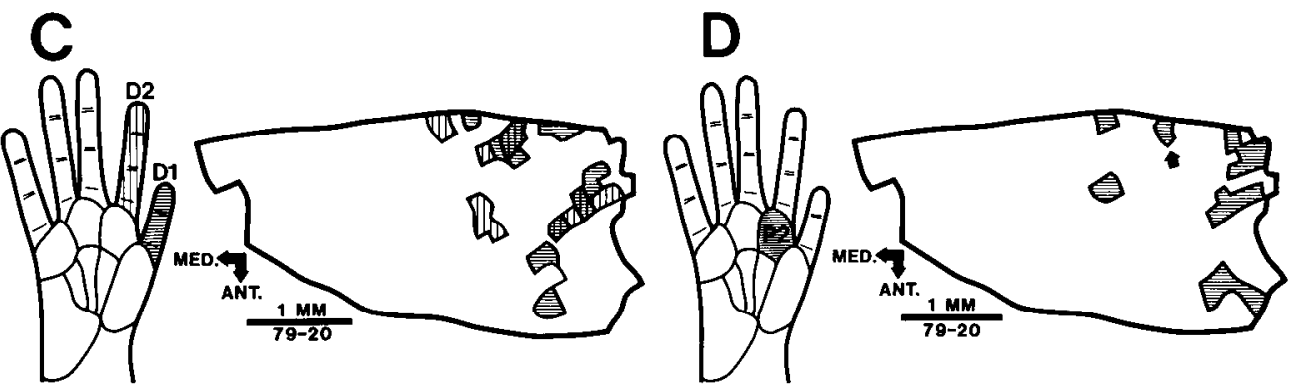
Figure 7. $C$, Patchlike representations of glabrous skin on DI (horizontal hatching) and D2 (vertical hatching). Note the overlap (horizontal and vertical hatching) in these representations resulting from MRF penetrations with field components on $D I$ and $D 2 . D$, Patchlike representation of $P 2$. Arrow indicates a patch that occupies a relatively normal location in the hand representation. Other conventions as in Figures 7 and 8.

cortex and were larger than normal (compare, e.g., P3 or hairy D1, D2, and D3 in Figs. $1 B$ and 5; Fig. 6B). The representations of these same skin areas enlarge in a similar fashion after transection and ligation of the median nerve (see Fig. $1 C$, Merzenich et al., 1983a, b). Thus, it appears certain that these enlargements in monkeys with repaired nerves resulted directly from the transection injury and were independent of median nerve regeneration.

These results suggest three conclusions: (1) Nerve transection and regeneration result in two major stages of cortical reorganization. Initial changes result directly from the transection injury. Following this stage, cortex undergoes further reorganization subsequent to nerve regeneration. (2) Cortical changes that directly result from nerve injury can persist after regeneration and, as a consequence, cortical organization after nerve repair represents a "montage" of the combined effects of injury and regeneration. (3) The effects of median nerve transection, repair, and regeneration are specifically restricted to parts of area $3 b$ that normally receive inputs from the median nerve.

\section{Topography in the cortical representations of reinnervated skin}

A unique pattern of topographical organization was reestablished in each monkey in cortical zones that were reactivated by regenerated inputs. At the same time, however, it appeared that several organizational features were common to all monkcys, and that individual differences reflected variability in the particular intermixture of these features. In this regard, reinnervated skin representations in all monkeys with repaired nerves were consistently different from representations of normally innervated skin in three major ways: (1) skin regions were represented at inappropriate locations in cortex, (2) representations of nonadjacent skin regions overlapped extensively, and (3) adjacent skin locations were represented in separate, discontinuous patches of cortex. For example, the representation of reinnervated pad skin on the radial side of the palm occupied two or more patchlike regions, which were widely distributed across the anterior-posterior extent of the hand area (Figs. $7 \mathrm{~B}, 8 \mathrm{~A}, 9 \mathrm{~A}$ ) whereas normally, this skin is represented in a continuous strip along the posterior margin of the hand cortex (e.g., Figs. $1 B$ and $7 A$ ). The representation of reinnervated glabrous skin on the radial digits was caudally displaced from its normal rostral location in a complementary manner (compare Fig. $7 \mathrm{~A}$ with $7 \mathrm{C}$, $8 B$, and $9 B$ ). Compared to the palm and digit representations of normal monkeys (e.g., Fig. $7 A$ ), reinnervated palm and digit representations overlapped extensively because of the high incidence of MRF sites with fields on both pads and digits (compare Fig. $7, B$ and $C, 8, A$ and $B$, and $9, A$ and $B$ ). Similarly the small, adjacent skin surfaces on individual pads or digits were also represented in fragmented patches (Figs. 7D, 8, $C$ and $D$, and $9 D$ ), which overlapped with representations of other pad or digital representations because of MRF sites (Fig. 9C), and which occupied abnormal locations in the hand representation (Figs. $1 B, 7 D, 8, C$ and $D, 9, C$ and $D$ ). The particular patchlike patterns varied widely in different monkeys. This can be seen, for example, by comparing the representations of $\mathrm{P} 2$ in Figures $8 D$ and $9 D, D 1$ in Figures $8 C$ and $9 C$, and D2 in Figures $8 C$ and $9 C$. From these findings, it is clear that transection and regeneration of the median nerve caused topographical remodeling in the area $3 \mathrm{~b}$ pad and digit representations.

In addition to remodeling changes, there were indications that more "normal" organizational features were also reestablished. First as previously described, many penetrations had a single cutaneous receptive field on a restricted part of the reinnervated skin. Thus, with respect to receptive field organization, fields at these cortical sites were similar to fields in area $3 \mathrm{~b}$ of normal monkeys, or to fields at sites representing the normally innervated side of the hand. A second normal feature was that patchlike representations of reinnervated pads and digits were sometimes reestablished at normal locations in the hand representation. This can be seen, for example, by comparing the locations of cortical regions representing glabrous D1 in a representative normal monkey (see Fig. $1 B$ ) with a monkey with a repaired nerve (Fig. $8 C$ ). The large patch (arrow) representing D1 in Figure $8 C$ appears to be in the neighborhood of its normal position whereas, by comparison, the remaining two small D1 patchcs appcar to bc abnormally located. Further instances of patchlike representations near their normal locations are shown for the representations of $\mathrm{P} 2$ (compare Figs. $1 B, 8 D$, and $9 D$ ) and $\mathrm{Pl}-\mathrm{TH}$ (compare Figs. $1 B$ and $7 D$ ). All monkeys with repaired nerves presented similar examples. This suggests that, after regeneration of a nerve subserving a skin area on the order of size of the median nerve skin territory, there is some possibility, perhaps attributable to chance peripheral reinnervation, that representations of reinnervated pads or digits will be reestablished near their normal, preinjury cortical locations.

A major contributing factor to the topographical disorganization in reinnervated skin representations was abnormal convergence of cutaneous inputs from multiple receptive fields at MRF sites. A detailed analysis of the receptive fields at MRF 


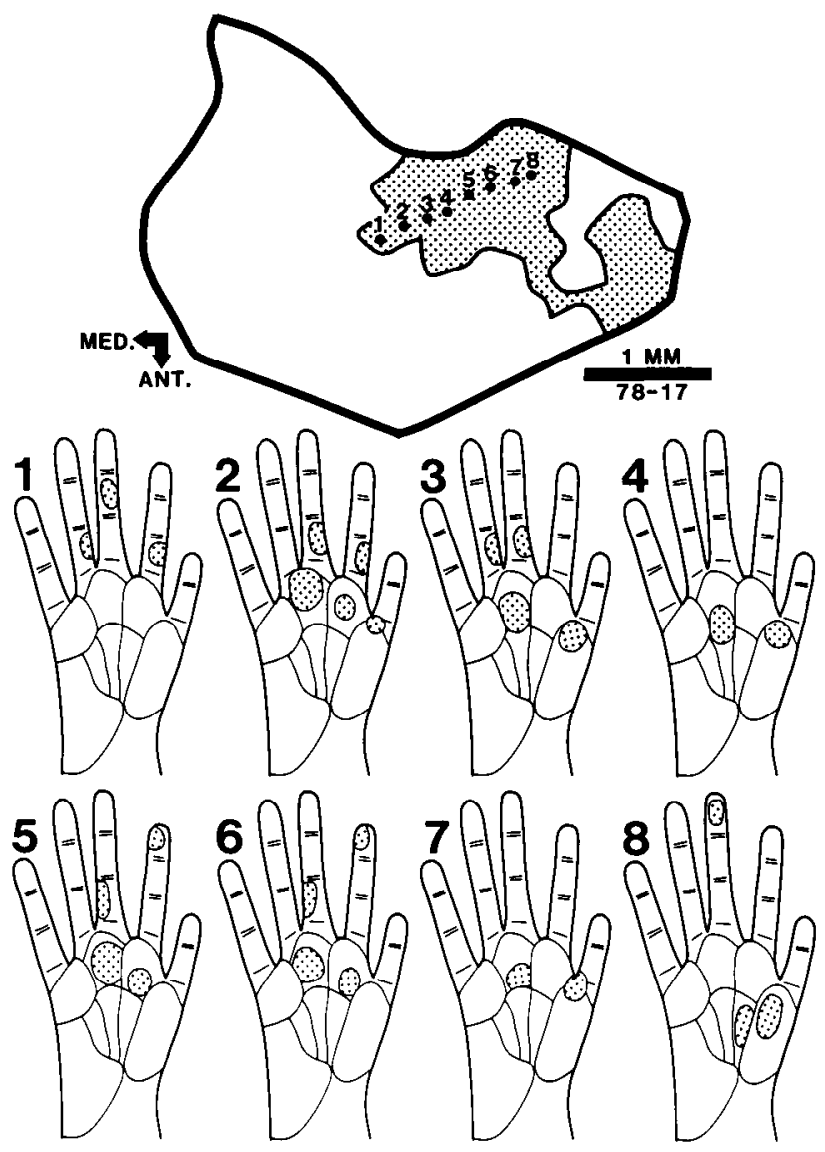

Figure 10. Overlap in receptive field components at adjacent MRF penetrations in area $3 \mathrm{~b}$. Top, The stippled region indicates parts of the hand representation that received median nerve inputs. Points $1-8$ indicate examples of adjacent cortical recording sites at which multiple cutaneous fields were defined. Bottom, Receptive fields defined at each of the 8 recording sites shown above. Note that at least one of the field components at adjacent sites overlaps to some degree.

sites suggested that, although receptive field organization was abnormal, it was neither diffuse nor completely random. As described previously, MRF sites had small continuous field components and, at any single site, component receptive fields were located on different pad and digit surfaces. Thus, receptive fields were spatially delimited and discretely distributed. In addition, further analyses indicated that MRF sites that occupied adjacent cortical positions sometimes had one or more component receptive fields, which overlapped to some degree (e.g., Fig. 10). As a consequence, cortical movements of several hundred microns were sometimes required before a completely different combination of fields was observed. In many instances, overlapping field components formed topographically continuous cortical representations of reinnervated skin areas (Fig. 11). The skin and corresponding cortical areas that had these "local" topographic relationships were small and usually coexisted with less ordered relationships (Fig. 11). Nevertheless, this local topography is interesting in its resemblance to the partially shifted receptive field overlap seen at adjacent penetrations in the area $3 \mathrm{~b}$ hand representation of normal monkeys. When considered together, small receptive field sizes, spatially discrete component fields at MRF sites, and local topographic relationships at adjacent sites indicate that regenerated inputs reactivated cortical neurons in a discrete, rather than diffuse, manner.

A final organizational feature of reinnervated skin representations appears relevant for understanding cortical reactivation

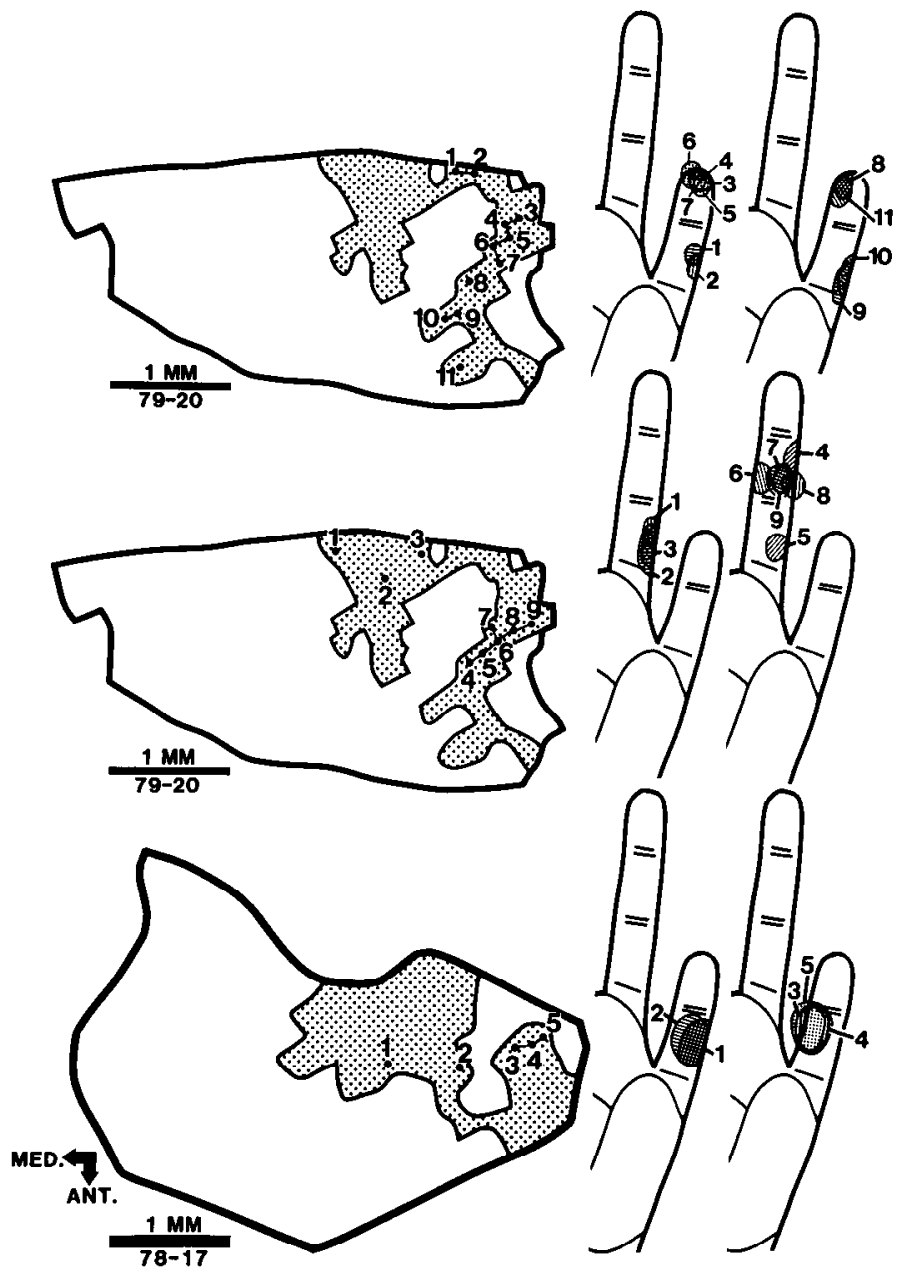

Figure 11. Topographical shifts in regenerated inputs to area $3 \mathrm{~b}$. Adjacent cortical sites sometimes represent inputs from adjacent or overlapping skin locations. Examples are (top) sites $I$ and 2,3-7, 9 and 10; (middle) sites 6-9; (bottom) sites 3-5. This type of organization is similar to the partially shifted overlap seen in normal monkeys. In other instances, adjacent cortical sites represent nonadjacent skin locations: Top, sites 8 and 9; middle, sites 4-6. Alternatively, nonadjacent cortical sites sometimes had overlapping fields: Top, sites 1 and $10,3,8$, and $I 1$; middle, sites $1-3 ;$ bottom, sites 1,2 , and 5 .

after nerve regeneration. As was discussed previously, chronic transection injury directly results in a loss of cutaneous fields on the median nerve skin territory and cortical recovery of substitute cutaneous fields from skin regions bordering the denervated zone (e.g., P3 and hairy D1-3). Regeneration resulted in subsequent replacement of substitute cutaneous fields with fields from one or more locations on the reinnervated skin. In the median nerve cortical zone, regeneration- vs injury-related changes in cutaneous receptive fields can be identified by field location in or out of the median nerve skin territory. At many MRF cortical sites, all receptive field components were well within the median nerve skin territory, indicating that all fields were attributable to regenerated inputs. Other MRF sites, however, had one or more receptive field components well within the median nerve territory but, in addition, had one field that extended onto one of the bordering skin regions, which had enlarged representations after transection injury (Fig. 12). The latter MRF sites appeared to reflect a mixture of fields resulting from both regeneration and nerve injury; that is, these sites had a receptive field organization which was "intermediate" between an injury-related single field on bordering skin and one or more regeneration-related fields on reinnervated skin. The field com- 


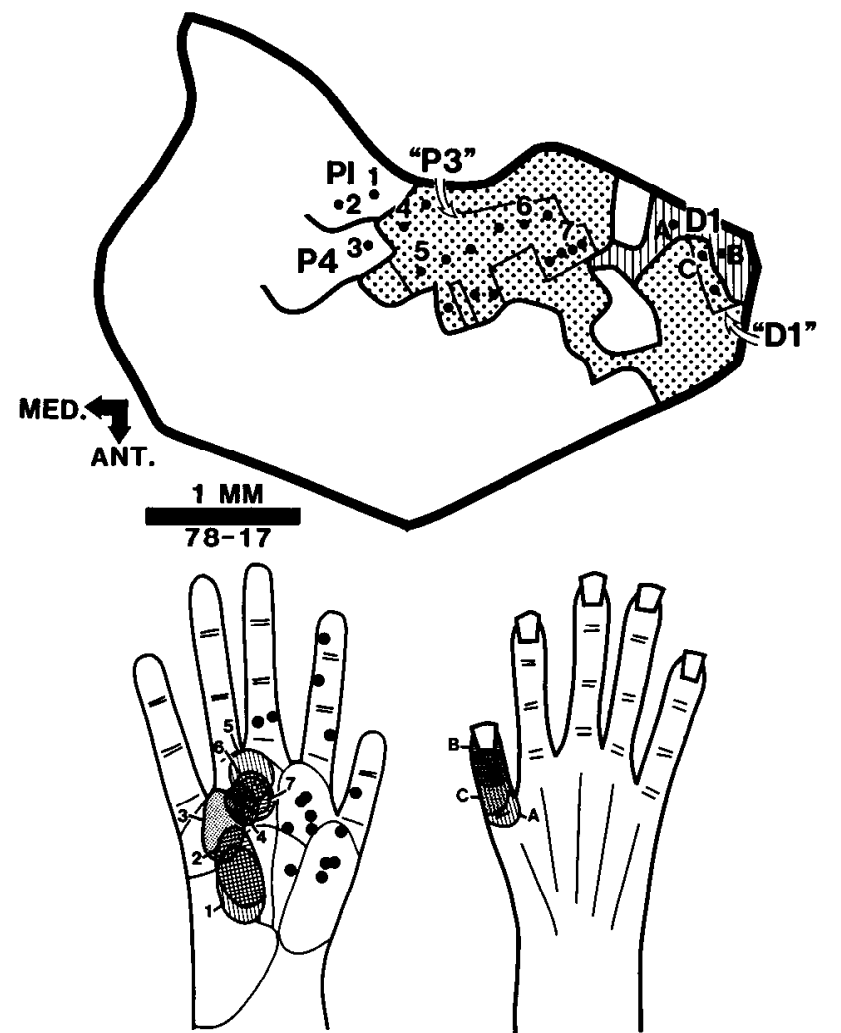

Figure 12. Injury- and regeneration-related "intermediate" field organization in area $3 \mathrm{~b}$. Sites $1-3(t o p)$ had single, normally appearing receptive fields on palmar pads $P I$ and $P 4$ (fields $1-3$, bottom left). Sites 4-7 and other nearby cortical sites (top, unnumbered large dots) each had one receptive field on palmar pad $P 3$, which was shifted in an overlapping fashion from the fields seen at sites $1-3$ (bottom, e.g., fields $4-7$ ). Thus, there was a representation of $P 3$ (top, thin border) that was topographically continuous with other palmar pads on the normally innervated side of the hand. This $P 3$ representation was similar to the expanded $P 3$ representation produced by median nerve transection injury (e.g., Fig. 1C). Sites 4-7 and the other unnumbered sites were MRF sites, however, and also had one or more other fields scattered across reinnervated skin (bottom, dots show field centers). The cutaneous field organization of these sites thus appears to be a composite of an "injuryrelated" field from bordering skin and "regeneration-related" fields from reinnervated skin. A similar but more limited example involving bordering skin on hairy $D I$ is also shown $(A, B, C$ 's top and bottom). Stippled region, the cortical zone representing regenerated inputs.

ponent on the bordering skin was usually topographically related to receptive fields at adjacent or nearby cortical sites representing other normally innervated skin surfaces (Fig. 12). This topographical continuity with normally innervated parts of the hand resembles the topographical organization in enlarged representations of bordering skin after chronic transection injury. These observations suggest that the reestablishment of regenerated inputs to cortical neurons involves functional interactions between regenerated and other inputs. The implication of this finding is that prior to becoming fully reactivated by regenerated inputs, area $3 b$ neurons may proceed through a transitional stage where activation is shared by regenerated and injury-induced substitute inputs.

\section{Cortical changes at different times after nerve repair}

Since regeneration requires new axonal growth from the point of injury, reinnervation proceeds largely in a proximal-to-distal fashion across the skin. Nerve regeneration rates have becn reported to vary from $1-4 \mathrm{~mm} / \mathrm{d}$ (Sunderland, 1978). In the present studies, the median nerve was repaired approximately

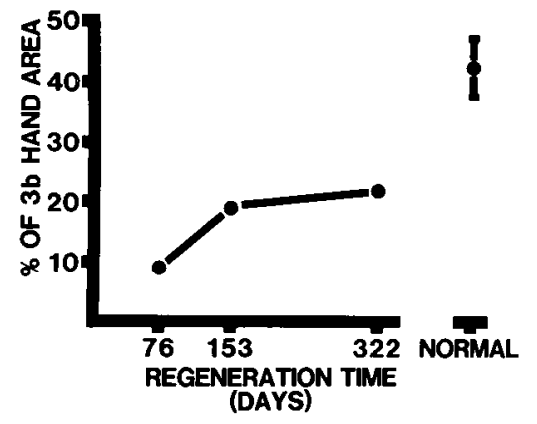

Figure 13. Increase in the size of the cortical area representing median nerve inputs in area $3 \mathrm{~b}$ as a function of postrepair time. The mean $( \pm \mathrm{SD})$ area representing median nerve inputs in normal monkeys is also shown.

$60-70 \mathrm{~mm}$ from the tip of the longest denervated digit. By a conservative estimate, regenerated axons would be expected in the distal digits 60-70 $\mathrm{d}$ after injury. The regeneration periods in the present experiments ranged from 76 to $322 \mathrm{~d}$. To study cortical changes over time, data from each monkey were analyzed separately and compared, either to data from other individual monkeys with different regeneration times, or mean data from the group of normal monkeys. Although data at different postrepair times were limited to results from a single animal, they provided useful preliminary observations on the nature of progressive cortical reorganizational changes after regeneration.

Changes in the size of cortical representations. The area of cortex activated by regenerated inputs from the median nerve increased in size as a function of postrepair time (Fig. 13). The monkey studied $153 \mathrm{~d}$ after nerve repair had a cortical representation occupying about $20 \%$ of the $3 \mathrm{~b}$ hand area, whereas the representation of the monkey studied at $76 \mathrm{~d}$ was less than half this size. The median nerve representation in the monkey studied at $322 \mathrm{~d}$ was modestly larger than the representation of the $153 \mathrm{~d}$ monkey. Even after $322 \mathrm{~d}$, however, the cortical area was still only half the size of the median nerve representation in normal monkeys (Fig. 13). During this period, the median nerve skin became more completely represented, as seen, for example, by comparing the density and distribution of reinnervated field centers at these three times (e.g., Fig. 3). These observations indicate that reclamation of cortical space by regenerated inputs continues over a time period two or more times greater than that required for initial reinnervation of distal skin.

A consideration of individual pad and digit representations provided a more detailed assessment of organizational changes at these postrepair times. In evaluating pad and digit representations, it was useful to consider reinnervated and normally innervated skin surfaces separately.

With respect to representations of reinnervated pads and digits, two changes were apparent. (1) Cortical recovery was related to the proximal-distal location of the reinnervated skin. For example, representations of proximally located pads were larger at $153 \mathrm{~d}$ after repair than at $76 \mathrm{~d}$, but were subsequently modestly smaller at $322 \mathrm{~d}(\mathrm{P} 1-\mathrm{TH}$ and $\mathrm{P} 2$; Fig. 14A). In contrast, the representations of reinnervated digits continuously increased but in a more gradual manner (D1 and D2 Glab.; Fig. 14A). At 153 and $322 \mathrm{~d}$, regenerated $\mathrm{P} 1-\mathrm{TH}$ and $\mathrm{P} 2$ inputs reactivated cortical areas that were somewhat larger than normal, whereas D1 and D2 representations were smaller than normal at all times (Fig. 14A). These findings indicate that the rate and extent of cortical reactivation from digital skin on more distal parts of the hand was more limited than the reactivation from morc proximal palmar surfaces. (2) The time-related increases in the total size of representations of reinnervated skin surfaces involved changes both in the number of cortical patches 

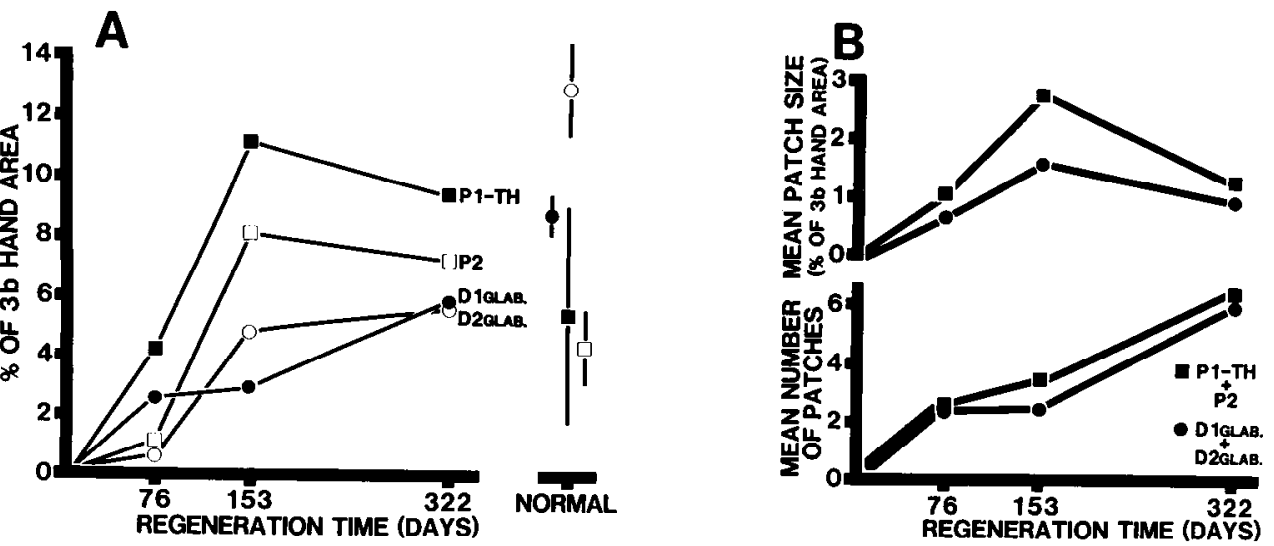

Figure 14. Changes in the size of individual pad and digit representations in area $3 \mathrm{~b}$ as a function of regeneration time. The representations of reinnervated skin parts $(A$ and $B)$ and normally innervated skin parts that bordered $(C)$ or did not border $(D)$ the reinnervated skin showed different time-related adjustments with respect to mean $( \pm S D)$ values from normal monkeys. Note that because of penetrations with multiple receptive fields, cortical representations of reinnervated pads or digits sometimes overlapped. The indicated measures show the total cortical area activated from the indicated skin, regardless of the degree of overlap with other representations.
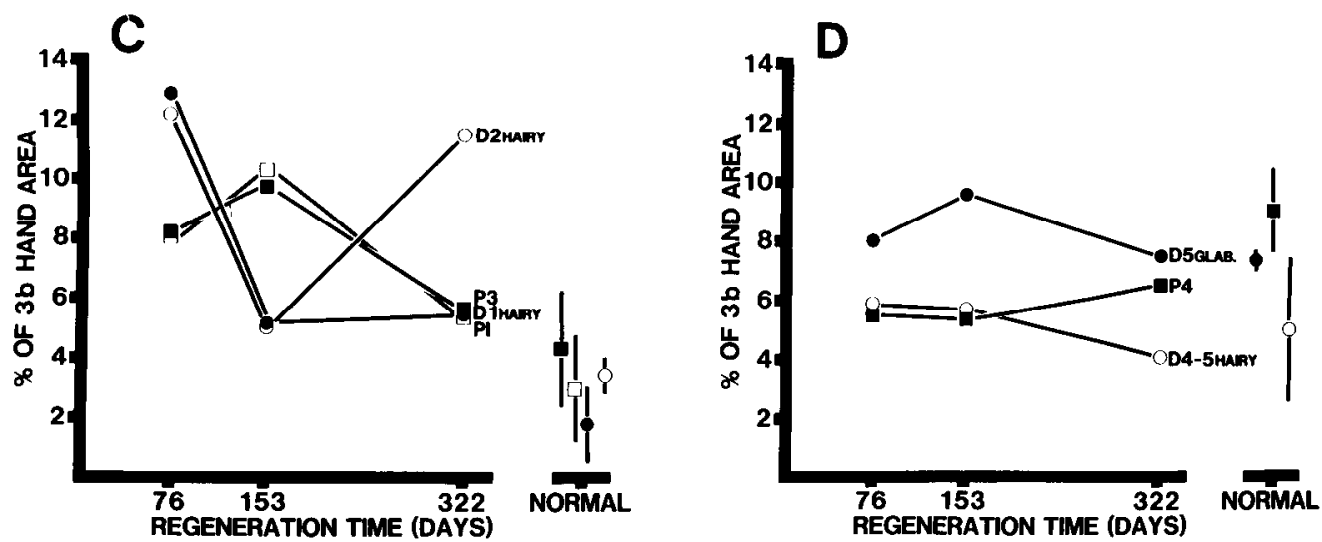

and the size of each patch. For example, the mean number of cortical patches at which reinnervated pads or digits were represented increased as a function of time after repair (Fig. 14B, bottom). The mean size per patch also increased at 76 and 153 $\mathrm{d}$, but decreased somewhat at $322 \mathrm{~d}$ (Fig. 14B, top). These observations suggest that separate, patchlike representations emerge and simultaneously enlarge during recovery. Over the time periods studied, enlargement and coalescence of patches is limited and, as a consequence, spatially dispersed cortical patches appear to be chronically maintained.

Changes in the representations of normally innervated pads and digits at different postrepair times were quite different from the above changes in reinnervated pad and digit representations. For example, representations of skin regions that bordered the reinnervated skin were consistently larger than normal at all regeneration times (e.g., D1 and 2 hairy, P3, and PI; Fig. 14C). Comparison of sizes at 322 vs $76 \mathrm{~d}$ further suggests that most of these representations were decreasing toward normal levels; however, the rate of change was variable, as can be seen by considering the sizes at $153 \mathrm{~d}$ (Fig. 14C). In contrast to those of bordering skin, representations of normally innervated pads and digits not bordering the median nerve skin remained more constant as a function of regeneration time, and were similar to normal in size (e.g., D5 Glab., D4 and 5 hairy, and, to a lesser extent, P4 in Fig. 14D). These findings suggest that the cortical representations of bordering skin regions were progressively altered in size during the first several months after repair, and that the cortical area gained by regenerated inputs was selectively reclaimed from representations of bordering skin regions that enlarged after injury.

Properties of MRF sites. Abnormal cutaneous field properties of neurons at MRF sites did not appear to undergo major changes over the times studied. For example, at all postrepair times, MRF penetrations comprised a high and relatively constant percentage of penetrations with regenerated inputs (Fig. 15, bottom). The mean number of fields observed at individual MRF sites did not systematically change (Fig. 15, middle), nor did the mean skin distance separating receptive field centers at MRF sites (Fig. 15, top). These findings indicate that the abnormal convergence of tactile information from spatially separate receptive field components to MRF sites was not eliminated during the time periods studied in these monkeys.

\section{Functional organization in area 1}

In two monkeys in which area $3 \mathrm{~b}$ was studied, complete maps were also made of the hand representation in area 1 . The area 1 hand representation is located immediately caudal to that of area $3 \mathrm{~b}$ (Fig. $1 \mathrm{~A}$ and Fig. 16, left) and is normally topographically organized in a rough mirror-image fashion with respect to area 3b (Figs. $1 B$ and 16, left; Merzenich et al., 1978). Area 1 maps provided an independent assessment of the cortical consequences of median nerve regeneration and allowed changes in cortical areas $3 b$ and 1 of the same monkey to be directly compared.

\section{Response properties of neurons}

The responses of area 1 neurons were generally similar to those of area $3 \mathrm{~b}$. In medial parts of area 1 , where normally innervated skin was represented, neurons had single, low-threshold cutaneous fields. In contrast, other, more lateral, parts of the hand representation contained neurons with multiple or single cutaneous fields on reinnervated skin, pacinian-like responses, or weak responses to tactile stimulation. Neurons with pacinianlike responses were encountered in a small percentage of penetrations in area 1 of normal monkeys (e.g., $3 \%[3 / 105]$ in the normal monkey in Fig. 16, left), and hence were not necessarily abnormal. One monkey with a repaired nerve (Fig. 16, right), however, had a somewhat larger than normal percentage of 

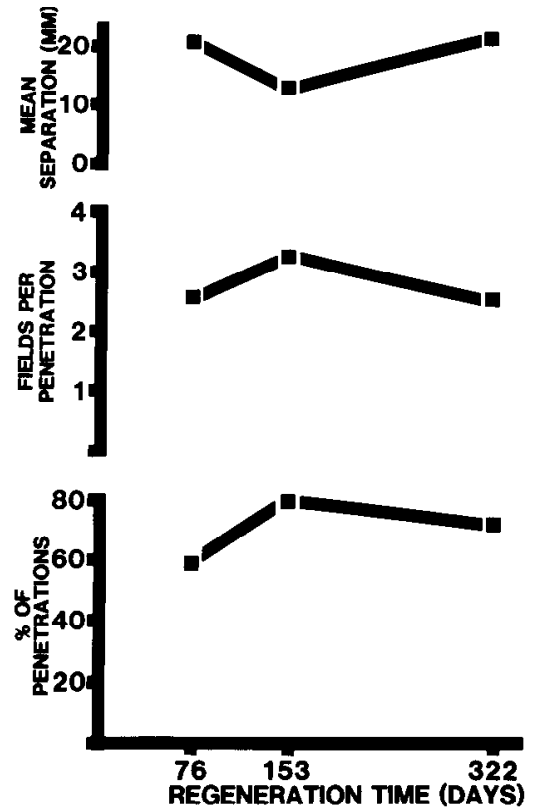

Figure 15. Persistence of abnormal cutaneous inputs in area $3 \mathrm{~b}$ as a function of regeneration time. Bottom, MRF sites comprised a consistently high proportion of cortical sites with regenerated inputs at all regeneration times. Middle, Mean number of fields per MRF site did not appear to change systematically over time. Top, Mean skin distances separating the centers of receptive fields at individual MRF sites did not systematically change.

pacinian-like response sites $(18 \%, 14 / 76)$, so it is possible that regeneration increased the incidence of these responses in area 1. Excluding pacinian-like sites, $65 \%(69 / 106)$ of the recording sites with regenerated inputs in area 1 were SRF sites, while $19 \%(20 / 106)$ were MRF, and $16 \%(17 / 106)$ were weakly responsive. While a large proportion of MRF sites had two to three fields, $35 \%(7 / 20)$ of these sites had five or more, a characteristic seen in only $3 \%(3 / 88)$ of the multiple field penetrations in area $3 \mathrm{~b}$ (Fig. 4, right). Similar to area $3 \mathrm{~b}$, the mean sizes of reinnervated fields at SRF and MRF sites in area 1 tended to be smaller (SRF: 48\%; MRF: 77\%) than the mean size of fields on normally innervated glabrous skin.

These results suggest that neurons in areas 1 and $3 \mathrm{~b}$ undergo similar types of response alterations after regeneration. The smaller proportion of MRF sites in area 1, as compared to area $3 b$, and the larger number of fields at about a third of the area
1 MRF sites, suggest that there are differences in the convergence of tactile information from regenerated inputs to neurons in these two cortical representations.

\section{Topographical organization}

The topography in the area 1 hand representation of monkeys with regenerated nerves can be summarized as follows: (1) Alterations in organization were mainly located in central to lateral parts of the hand representation, while organization in the more medial hand cortex was more normal (Fig. 16, right). (2) In contrast to normal organization (e.g., Fig. 16, left), hairy skin regions bordering reinnervated skin were represented in large cortical zones along the lateral and caudal margins of area 1 (e.g., D1-3 and $\mathrm{H}$ in Fig. 16, right). This pattern of representation resembles the organization seen after chronic median nerve transection (Merzenich et al., 1983a) and presumably resulted from median nerve injury. (3) Representations of reinnervated skin regions were topographically abnormal in that adjacent skin locations were represented in discontinuous patches, and representations of nonadjacent skin regions commonly overlapped (Fig. 17). (4) Patchlike representations of reinnervated pads and digits occupied both normal and abnormal locations in the hand representation (compare P1-TH in Fig. 16, left and Fig. 17). (5) Some adjacent MRF sites had overlapping receptive field components. (6) Some MRF sites had one receptive field on skin bordering the reinnervated zone and one or more fields on reinnervated skin; thus, as described for area $3 b$, these sites appeared to have a receptive field structure "intermediate" between postinjury- and regeneration-related organizations. These findings indicate that the general sequence of reorganizational adjustments in area 1 was similar to that seen in area $3 \mathrm{~b}$.

Further analysis also showed differences in the topographical consequences of nerve repair in areas 1 and $3 \mathrm{~b}$. Major among these was that patchlike representations of reinnervated skin regions in areas 1 and $3 b$ of the same monkey were different in the ways they were abnormal. For example, the area 1 representation of reinnervated P2 in the monkey shown in Figure 17 consisted of two widely separated patches of cortex, whereas P2 inputs in area $3 \mathrm{~b}$ were represented in five separate patches (Fig. 17 , left). Both area 1 and $3 \mathrm{~b}$ patterns of $\mathrm{P} 2$ representation were abnormal (compare Fig. 17 with Figs. 16 and $1 B$ ) and, in addition, the transformations from normal were different in each cortical area. This also holds for the representations of P1-TH, glabrous D1, and glabrous D2 (Fig. 17). It can also be seen that abnormal patterns of overlap of representations were different in areas 1 and $3 b$ (compare overlap zones of P1-TH with P2 and D1 with D2 in Fig. 17). If abnormal patterns of cortical

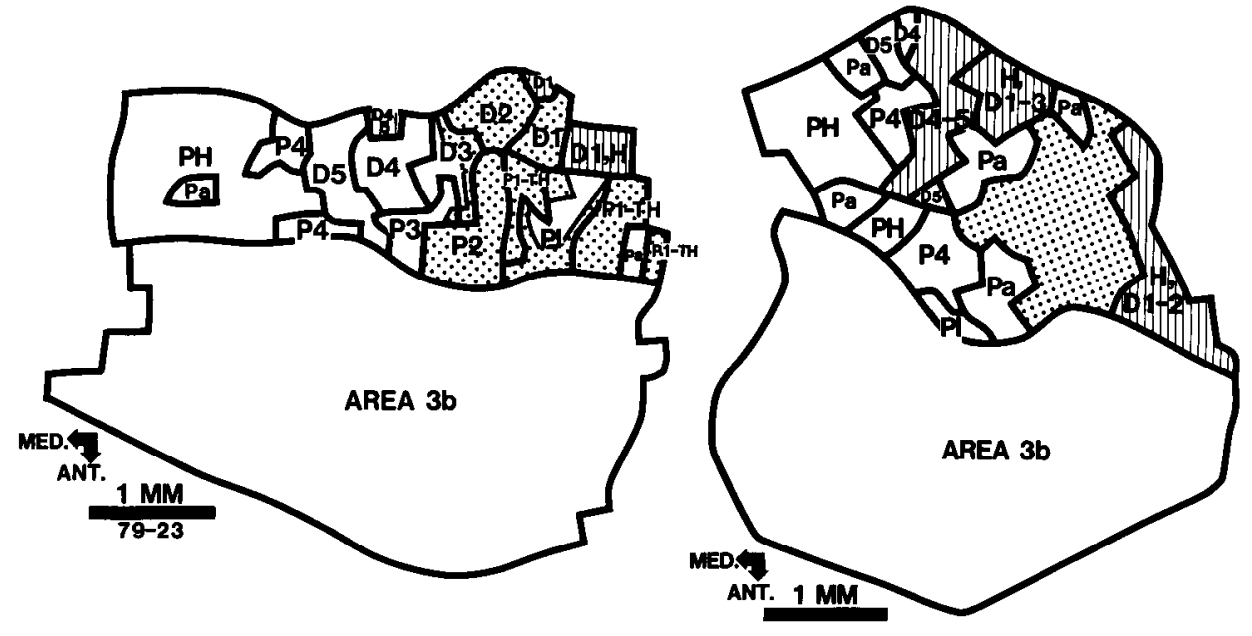

Figure 16. Representation of the hand in area 1. Left, Normal owl monkey. Cutaneous inputs from the glabrous (unhatched and stippled) and hairy (hatched) hand surfaces are represented in a rough mirror-image fashion with respect to the more rostrally located hand representation in area $3 \mathrm{~b}$ (see Figure 1B). Stippling, Cortex with median nerve inputs. $P a$, Cortex with pacinian-like inputs. Right, Area 1 in a monkey with a repaired median nerve. Conventions same as for normal monkey. 
Figure 17. Representations of reinnervated pads (left) and digits (right) in areas 1 and $3 \mathrm{~b}$ of the same monkey. Representations in both areas are highly abnormal (e.g., compare with the representations of the normal monkey in Figs. $1 B$ and 16, left). Note: The overlap (horizontal and vertical hatching) in the representations of individual pads and digits resulting from MRF penetrations, and the transformations from the normal pattern of representation are different in each area. As a consequence, the mirrorimage pattern normally resulting in areas 1 and $3 b$ from inputs of, for example, a digit is no longer apparent.
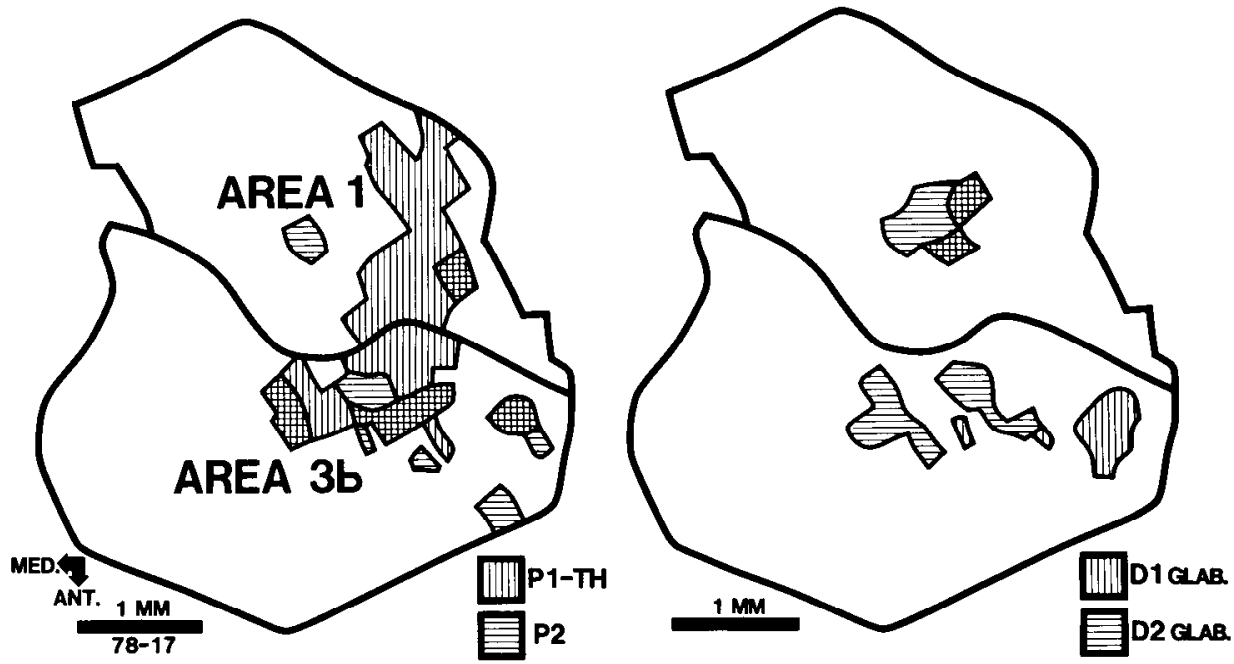

representation were completely due to peripheral changes in the regenerated median nerve, similar transformations from normal might be expected in both cortical representations. That is clearly not the case.

\section{Discussion}

\section{Present results}

First-order neurons in the somatosensory system of adult mammals retain a lifelong ability to regenerate new distal processes. In the present experiments, we used this ability to study the effects of regrowth of new sensory connections on functional organization in somatosensory cortical areas $3 \mathrm{~b}$ and 1 of monkeys. The general finding was that peripheral regeneration resulted in a reactivation of central circuits and, as a consequence, cortical neurons recovered their capacity to process inputs from previously denervated regions of skin. Cortical recovery in these adult primates was characterized by the following features.

Sequential reorganization. Cortical representations of reinnervated skin regions were recovered via a sequential process entailing at least two stages of reorganization. The first stage occurred as a direct result of the transection injury and involved a loss of the cortical representations of denervated skin surfaces and a corresponding enlargement of the representations of adjacent skin surfaces into cortical regions that previously represented the denervated skin. A second stage of reorganization occurred as a result of subsequent nerve regeneration. During this stage, new cortical representations of the reinnervated skin displaced the enlarged representations that had been established after injury. The transition from the first to the second stage of reorganization appears to occur in a progressive manner, since cortical neurons sometimes have a receptive field organization that appears to result from an interaction of injury- and regeneration-related inputs.

Prolonged time course. The cortical effects of the transection injury were highly persistent and, even as long as 11 months after repair, cortical organization reflected the combined effects of both the transection injury and subsequent regeneration. Because of continued adjustments after reinnervation or slow reinnervation, the overall size of the cortical area representing regenerated inputs in area $3 \mathrm{~b}$ continued to enlarge over regeneration periods two or more times longer than were required for the earliest reinnervation. Proximal skin locations in the reinnervated zone had a faster and more extensive recovery of cortical space than did distal skin locations.

Involvement of spatially delimited zones of the skin and cortex. During recovery, reorganization primarily involved cortical zones that normally represented inputs from the injured nerve and skin zones in or adjacent to the denervated region. Normal functional organization was maintained in neighboring parts of cortex, which continued to receive normal inputs from hand regions not adjacent to the denervated zone.

Alterations of neuronal responses. Recovery resulted in qualitative changes in the responses of some cortical neurons to tactile stimuli. In contrast to the normal response to a single, small, cutaneous receptive field, responses after regeneration were more variable. In addition to single, small cutaneous fields, cortical sites had multiple cutaneous fields, large pacinian-like fields, or little or no apparent response to tactile stimulation. As exemplified by the receptive field organization at multiple receptive field sites, these response alterations were persistent.

Extensive topographical remodeling. Recovery entailed significant changes in the topographical organization of cortical representations of reinnervated skin surfaces. Although idiosyncratic topographical patterns were reestablished in each case, the general types of alterations contributing to individual differences included (1) reestablishment of discontinuous, patchlike representations, (2) recovery of representations at correct, as well as incorrect, locations, and (3) extensive overlap of representations of nonadjacent, reinnervated surfaces.

Reestablishment of discrete functional features. Although cortical reactivation was abnormal in many respects, it was not diffuse or unstructured. The receptive field organization of cortical neurons that recovered responsiveness to regenerated inputs was discrete, as reflected, for example, by the recovery of small cutaneous receptive fields and spatially distinct component fields at multiple receptive field sites. In addition, neurons at adjacent cortical sites overlapped to varying degrees and, as a consequence, topographical representations of reinnervated skin surfaces were reestablished in localized areas of cortex.

Different alterations in separate subdivisions of cortex. Within an individual monkey, the patchlike representations of reinnervated pads and digits were transformed from normal in different ways within cortical areas $3 \mathrm{~b}$ and 1 . As a result, there was a dissociation of the mirror-image topographical relationship that normally exists between these two areas.

These findings suggest that cortical recovery involves a dynamic reorganizational process that begins at injury and continues for many months after initial reestablishment of new inputs. The reestablishment of discrete cutaneous receptive fields and local topography suggests that some degree of selectivity is incorporated in the cortical recovery process; however, the functional impact of this selectivity is complicated by a concomitant emergence of aberrant features, such as multiple receptive fields, 
smaller than normal cortical areas representing regenerated inputs, and patchlike representations of reinnervated skin surfaces in abnormal cortical locations. We conclude that cortical recovery after nerve repair and regeneration results in a significant restoration of the tactile processing capacities of cortex but is unlikely to result in widespread reestablishment of normal patterns of functional organization.

\section{Previous studies on the central consequences of nerve regeneration}

There have been very few studies on the cortical effects of nerve transection and regeneration. In the only other study in primates, Paul ct al. (1972) found that the functional organization of cortical areas $3 \mathrm{~b}$ and 1 of adult macaque monkeys was substantially changed after combined repair of the median, ulnar, and radial nerves to the hand. Cortical recovery was characterized by (1) alterations in neuronal responses to tactile stimuli including, for example, reestablishment of multiplc reccptive fields, (2) widespread topographical reorganization, and (3) different reorganizational changes in areas $3 b$ and 1 . Waite and Cragg (1982) studied the S-I cortex of adult rats that had undergone transection of the infraorbital nerve to the vibrissae. Following regeneration, the representation of reinnervated vibrissae inputs was smaller than normal, while the representations of normally innervated parts of the face were enlarged. In addition, cortical neurons had abnormal responses to stimulation of more than one vibrissa and the vibrissae representation was topographically disorganized. The findings from both these studies are in good agreement with the present results in indicating, first, that regeneration restores the ability of cortical neurons to respond to tactile stimulation of reinnervated skin and, second, that it commonly results in reestablishment of abnormal functional features in cortex.

The present findings are also consistent with the results of studies at subcortical levels of the somatosensory system. For example, abnormal receptive fields and abnormal patterns of topographical organization have been reported in the trigeminal nucleus of rats after transection and regeneration of the infraorbital nerve (Waite and Cragg, 1982) and in the dorsal horn of the spinal cord of cats after transection and regeneration of the saphenous and sciatic nerves (Lisney, 1983a). This suggests that the features of recovery seen in the present experiments reflect a general pattern of central recovery reiterated across all levels of the somatosensory system. The different topographical transformations in cortical areas $3 b$ and 1 also suggest some variability in processing of regenerated inputs in different central structures. Given this possibility, further studies are necessary to determine whether there are also detailed differences in the processing of information from regenerated inputs at cortical and subcortical levels of these monkeys.

The present findings also relate to a recent study on cortical recovery after crush and regeneration of the median nerve (Wall et al., 1983). Three salient points arise from a comparison of these studies. In both studies, cortical recovery involved a sequential reorganizational process during which cortical changes resulted from both the initial injury and subsequent regeneration. Also, cortical changes resulting from the initial injury were, in general, similar after transection and crush types of injury. For example, after both injuries there was a loss of cortical representation of denervated skin and a corresponding enlargement of the representations of normally innervated skin surfaces near the denervated zone. Finally, unlike the injury-related reorganizational stage, reorganization due to subsequent nerve regeneration is substantially different after transection and crush injuries. After nerve transection and regeneration, for example, multiple receptive fields are common and patchlike representations with limited local topography are often reestablished in abnormal cortical locations. However, after nerve crush and regeneration, single receptive fields were typically restored, and topographically organized representations of reinnervated skin surfaces were reestablished with a high degree of specificity in normal cortical locations. A similar recovery of normal topographical organization has been reported in the S-I cortex of rats after crush and regeneration of the infraorbital nerve (Waite and Cragg, 1982) and in the spinal cord dorsal horn of cats after crush and regeneration of the saphenous and sciatic nerves (Lisney, 1983b). Regenerated peripheral nerves typically undergo better functional recovery after nerve crush injury than after transection injury (Dykes and Terzis, 1979; Horch, 1979; Horch and Lisney, 1981; Terzis and Dykes, 1980). This suggests that the contrasting cortical recovery patterns after these different injuries are attributable, to some extent, to differences in the newly grown peripheral inputs.

\section{Peripheral vs central contributions to cortical recovery}

Peripheral receptor organs and sensory axons undergo a variety of alterations after nerve transection and regeneration. With regard to receptors, for example, Meissner's corpuscles and Merkel's cells can undergo degenerative changes after denervation (Brown and Iggo, 1963; Burgess et al., 1974; Dellon, 1981; Dellon et al., 1975; English, 1977; Wong and Kanagasuntheram, 1971). These changes can be reversed after subsequent reinnervation, provided denervation is not too prolonged (Brown and Iggo, 1963; Burgess et al., 1974; Dellon, 1981; Jabaley et al., 1976; Wong and Kanagasuntheram, 1971). By comparison, pacinian corpuscles remain largely unchanged for many months or years after denervation (Glees et al., 1949; Lee, 1936; Miller and Rusenas, 1976; Schiff and Loewenstein, 1972; Wong and Kanagasuntheram, 1971; Zelena, 1981). Peripheral nerves also undergo a variety of structural and functional changes, including (1) reductions in the numbers of cutaneous fibers due to sensory cell death (e.g., Cavanaugh, 1951; Ranson, 1906; Wall and Devor, 1978), entrapment of fibers at repair sites (Sunderland, 1978), or erroneous reinnervation of noncutaneous tissue (Karpati et al., 1981; Zalewski, 1970); (2) sprouting of regenerating axons into multiple branches that can reestablish cutaneous fields at discontinuous skin sites (Horch and Lisney, 1981; Mackel et al., 1983b; Terzis and Dykes, 1980); (3) decreases in axonal conduction velocity (Buchtal and Kuhl, 1979; Burgess and Horch, 1973; Cragg and Thomas, 1961; Hallin et al., 1981; Horch, 1976; Horch and I isney, 1981; Rosenfalck, 1980; Terzis, 1981; Terzis and Dykes, 1980); (4) alterations in topographical organization of nerves due to haphazard growth of regenerated axons to abnormal skin locations (Hallin et al., 1981; Horch, 1979; Terzis, 1981); (5) some cross-reinnervation of the wrong type of receptor organ by regenerated axons (Burgess and Horch, 1973; Burgess et al., 1974; Horch, 1976, 1979; Horch and Burgess, 1980; Schiff and Loewenstein, 1972); (6) reestablishment of cutaneous receptive fields that usually range in size from small to normal (Mackel et al., 1983a; Terzis and Dykes, 1980); and (7) reestablishment of elevated or normal tactile thresholds (Burgess and Horch, 1973; Hallin et al., 1981; Horch, 1976; Mackel et al., 1983a; Terzis and Dykes, 1980).

Given the probability that these peripheral changes occurred in the present study, it is not surprising that normal cortical organization was not restored. Consistent with this view, a number of cortical reorganizational features appear to be at least partly attributable to peripheral changes, including the following: (1) Multiple receptive field organization and abnormalities in cortical topographical organization are presumably related to haphazard reinnervation of the skin by regenerated axons. Growth of regenerated axons to inappropriate addresses probably reestablishes an abnormal convergence of spatial information onto subcortical central neurons which, in turn, is transmitted to cortex. (2) Pacinian-like responses are not observed in area $3 b$ of normal owl monkeys (Merzenich et al., 1978; Wall 
et al., 1983) or of owl monkeys with transected median nerves (Merzenich et al., 1983a, b). Their appearance following nerve regeneration suggests several possibilities, including altered functioning of reinnervated receptors and cross-reinnervation of pacinian corpuscles by sensory axons previously specialized differently. Other observations on pacinian corpuscles are consistent with the latter possibility (Schiff and Loewenstein, 1972). (3) The incomplete activation of cortical space by regenerated inputs is probably related to a reduction in the cutaneous sensory fiber population and to less effective spatial and temporal convergence of information caused by haphazard reinnervation and changes in the conduction velocity of regenerated axons.

Other cortical features do not appear to be adequately explained by peripheral factors. First, there was a greater degree of functional specificity in cortical organization than might be expected. This specificity was reflected in (1) recovery of small and distinct receptive fields, (2) spatial segregation of component fields at multiple field sites onto functionally discrete surfaces of pads and digits, (3) local topographical organization in cortical representations of reinnervated skin surfaces, and (4) reestablishment of overlapping field components at adjacent multiple field sites in cortex. All these features reflect a spatial orderliness that is not predictable from the haphazard and randomized pattern of peripheral reinnervation resulting from nerve transection and regeneration (Hallin et al., 1981; Horch, 1979; Terzis, 1981). A second reorganizational feature not explained by peripheral factors involved the different transformations in topographical organization in areas $3 \mathrm{~b}$ and 1 . If central pathways to cortex process peripheral inputs with no changes, identical transformations from normal might be expected in all cortical areas. This production of multiple patterns of cortical reorganization from one abnormally organized set of peripheral inputs suggests that cortical organization is not passively determined by peripheral inputs. Together, the above features of cortical reorganization would appear to reflect intrinsic mechanisms for some degree of central resorting of peripheral inputs after regeneration. Owing to the longstanding recognition of the peripheral effects of regeneration, it has usually been proposed that recovery depends entirely on successful regeneration of the transected nerve; consequently, the contribution of central factors to recovery has received little or no attention. From the above evidence, however, we conclude that cortical recovery depends on a combination of central and peripheral factors and that the CNS plays a more important role in recovery than was previously thought. This conclusion is consistent with recent studies indicating that central somatosensory structures of adult mammals maintain a lifelong capacity to functionally reorganize in response to loss of peripheral inputs (see Kaas et al., 1983, for a recent review).

\section{Sensory consequences of nerve transection and regeneration and possible relationships to cortical reorganization}

In humans, peripheral nerve injury per se results in loss of tactile sensations in skin regions normally innervated by the damaged nerve (Boring, 1916; Buchthal and Kuhl, 1979; Cobb, 1919; Franz, 1909; Head, 1920; Sharpey-Schafer, 1928; Trotter and Davies, 1909). In addition, tactile stimulation of skin areas near the margins of deafferented regions commonly evokes a variety of unusual sensations, such as hypersensitivity (for a recent review, see Wall and Kaas, 1985). Following median nerve transection and ligation in monkcys, cortical representations of denervated skin regions are lost, and cortical representations of skin regions on the margins of the denervated zone expand in size (Merzenich et al., 1983a, b). This suggests that there are relationships between the losses of tactile sensations and of cortical representations, as well as between unusual sensations from bordering skin and enlargements in cortical representations of these skin regions. Under typical conditions, in which regeneration requires several weeks or months, these injury-related sensory and cortical changes are established before regenerationrelated changes.

Tactile sensory recovery after subsequent nerve regeneration is highly dependent on the nature of the injury. In contrast to the good recovery likely after crush injury, nerve repair usually results in more limited recovery (Buchthal and Kuhl, 1979; Dellon, 1981; Seddon, 1943, Sharpey-Schafer, 1930; Stopford, 1926). Several types of sensory deficiencies are typically reported after nerve repair:

Tactile sensitivity is only partially recovered in some reinnervated skin regions. Loss of sensitivity is usually reflected in increased thresholds to von Frey hairs (Boring, 1916; Dellon, 1981; Head, 1920; Moberg, 1962; Onne, 1962; Poppen, 1980; Trotter and Davies, 1909; Tupper, 1980) and impaired 2-point discrimination (Boeke, 1940; Dellon, 1981; Flynn and Flynn, 1962; Ford and Woodhall, 1938; Head, 1920; Mackel et al., 1983a; Moberg, 1962; Omer, 1974; Onne, 1962; Stromberg et al., 1961).

Tactile stimulation of the reinnervated skin may produce hypersensitive reactions (Ford and Woodhall, 1938; Hallin et al., 1981; Head, 1920; Mackel et al., 1983a; Trotter and Davies, 1909). The particular sensation produced can vary from being vivid (hyperesthesia), to unpleasant (dysesthesia), to painful (hyperpathia).

Punctate tactile stimuli are poorly localized and commonly referred to locations other than the stimulated site (Ford and Woodhall, 1938; Hallin et al., 1981; Hawkins, 1948; Head, 1920; Langley, 1908; Mackel et al., 1983a; Mitchell, 1895; Sperry, 1945; Stopford, 1927; Trotter and Davies, 1909). Referred sensations can be perceived to be of variable strength, in small or diffuse skin regions, in one location or simultaneously in more than one location, or simultaneously in correct and incorrect locations.

The ability to identify patterns, forms, and objects is impaired (Dellon, 1981; Ford and Woodhall, 1938; Sperry, 1945). This loss of function presumably involves a dissociation of sensations that define spatial aspects of tactile stimuli, and is reflected in measures of diminished tactile recognition (Dellon, 1981; Mi1lesi, 1980; Moberg, 1962).

In what ways are these sensory changes and cortical reorganizational changes related? Several plausible associations might be suggested.

1. If loss of tactile sensation after nerve transection is related to loss of central representations of the deafferented skin, it is reasonable to further postulate that the partial recovery of tactile sensitivity commonly seen after nerve repair is related to the present finding that regenerated nerves may only reactivate a fraction of their normal cortical space.

2. The hypersensitivity that occurs after regeneration is similar to the hypersensitivity resulting directly from nerve injury except that, after injury, hypersensitivity is evoked from skin areas adjacent to the denervated zone whereas, following regeneration, it is evoked from reinnervated (previously denervated) skin, as well as from adjacent skin areas. As was discussed above, hypersensitivity after injury may be related to enlarged cortical representations of adjacent skin regions. If repaired and regenerated nerves reactivate only part of their normal cortical space, injury-related cortical enlargements can remain apparent after regeneration. Thus, the hypersensitivity around rcinncrvated skin areas after regeneration may be related to the persistence of these enlargements. Within the reinnervated skin itself, hypersensitivity may be related to abnormally large representations of regional patches of skin, like, for example, the enlargements in reinnervated pad representations seen in the present study. The assumption underlying these relationships is 
that the strength, or other qualities, of sensations is related to the size of the central cell population recruited during stimulation of specific skin zones.

3. False localization of tactile stimuli within reinnervated skin zones seems related to abnormal patterns of cortical topographical organization. This relationship has been previously suggested by Paul et al. (1972). Several correspondences between the present findings and human sensory findings are consistent with this view. (a) Following regeneration, topographical reorganization occurs specifically in the cortical region, where the reinnervated skin is represented. Similarly, sensory mislocalization is restricted specifically to reinnervated skin. (b) Reinnervated skin regions are represented in patchlike cortical representations, and a point on the skin is commonly represented in several cortical locations. Some of these cortical locations may have normally represented that skin location, while others may have represented different parts of the hand prior to nerve repair. Similarly, the sensation generated by a punctate stimulus to reinnervated skin is often simultaneously referred to several skin sites. The correct location may or may not be identified. (c) The cortical patches representing delimited reinnervated skin locations vary in size. Skin regions to which sensory mislocalizations are referred vary in size, and the strength of the referral varies. (d) Highly idiosyncratic patterns of cortical topography are produced after nerve repair in different animals. Similarly, the specific arrangement of erroneous and correctly localized stimuli is variable in different humans. Together, these observations suggest a direct relationship between degraded localization and cortical topography disorders after nerve repair.

4. Finally, tactile object recognition presumably requires that central circuits be supplied with an "image" of the stimulus. Under normal conditions, adjacent points on the skin of, for example, a digit tip are commonly represented at closely adjacent sites in cortex. Thus, information on spatial patterns of skin activation is probably contained in the pattern of activation produced across cortical positions. Following nerve repair, skinto-cortex adjacencies are abnormal. There are increases in convergence of information such that more than one nonadjacent skin location may activate a single cortical site and, conversely, a single skin location may be represented in several widely separated cortical positions. It is plausible that impaired object recognition is related to changes in patterns of cortical activation produced by alterations of normal adjacencies in skin-to-cortex circuits. A different, but equally interesting, possibility is that object recognition requires a highly specific integration of spatial information across different cortical areas, and that reestablishment of different topographical transformations, as seen, for example, in cortical areas $3 b$ and 1 , results in a degradation of this integrative capacity.

\section{Sensory corrections and central compensations in systems with regenerated inputs}

Sensory functions are clearly altered after nerve repair and, in many instances, tactile deficiencies remain apparent for years (Hallin et al., 1981; Mackel et al., 1983a; Sperry, 1945). Reviews of clinical investigations further suggest, however, that tactile recovery can be improved by sensory reeducation programs. For example, in a group of patients that had undergone reeducation after distal repair of the median nerve, Dellon (1981) reported that $50 \%$ recovered 2-point discrimination to an S4 level, and $38 \%$ recovered to an $\mathrm{S} 3+$ level $(\mathrm{S} 4=$ normal discrimination of 2-6 mm; S3+ = 7-15 mm), whereas in a group of patients that were not reeducated, none recovered to $\mathrm{S} 4$, and $24 \%$ recovered to $S 3+$. Dellon further reported that "virtually $100 \%$ correction" of mislocalization can occur after reeducation and that hypersensitive reactions from reinnervated skin regions can be diminished. Similarly, Wynn-Parry (1973) reported that false localization can be corrected; about $25 \%$ of his patients had near-normal 2-point discrimination after reeducation. Thus it appears that sensory improvements, especially the correction of false localization, are possible.

In the present study, no specific efforts were made to reeducate; however, as the animals used their hands for feeding and climbing, they may have experienced cumulative, use-related cortical effects as a function of postrepair recovery time. Although our data on recovery time are limited to observations from single monkeys, some features of cortical organization appeared to change at different postrepair times: In area $3 \mathrm{~b}$, the overall cortical area representing regenerated inputs changed in size, and patchlike representations of reinncrvated pads and digits changed in numbers of patches and the size of each patch. In contrast, other features remained more constant at all times after repair: The incidence of multiple receptive field sites did not change; the number of fields per multiple field site remained constant; and skin ficlds at individual multiple field sites remained discontinuous, with no sign of merging over time. These findings are interesting in that, following wrist-level repair of the median nerve in humans, reeducation results in the correction of faulty localization within 1-2 years (Dellon, 1981). Since the distance from the wrist to the fingertips in humans is about $3-4 \times$ the comparable distance in owl monkeys, complete reinnervation of the median nerve territory should take about 3$4 \times$ as long in humans. Thus, a $1-2$ year regeneration period should be roughly comparable to a 3-8 month period in these monkeys. This suggests that good tactile localization can occur in humans during a time period when, as suggested by the monkey data, considerable disorder still exists in cortex.

What does this suggest about the relationship between sensory localization improvements and cortical organization? If the reorganizational changes in the present study are representative of general changes in somatosensory structures important for tactile sensory localization, several hypotheses might be suggested.

First, the cortical disorder seen in monkeys may be similar to human disorder prior to reeducation. If appropriate sensory reeducation had been carried out on the monkeys in the present studies, cortical disorder might have become less evident. In this hypothesis, sensory improvements involve central compensations that normalize the abnormalities in cortical functions resulting after regeneration (Sperry, 1945).

A second hypothesis is that good tactile localization may be reestablished, even though cortical organization remains highly abnormal. Consistent with this view, a series of recent studies in owl monkeys have shown that normal cortical organization can be altered by nerve injury (Merzenich et al., 1983a, b), digit amputation (Merzenich et al., 1984), cortical lesions (Jenkins et al., 1982), and increased use of fingers (Jenkins et al., 1984). This strongly suggests that normal features of cortical organization (e.g., topographical order, cutaneous receptive field structure and size) are dynamically controlled by activity-dependent, sensory system filters, and that, within the framework of wider anatomical connections between skin and cortex, representations of skin areas can effectively be switched to different cortical positions without causing sensory localization impairments. With respect to the present studies, the implication of these findings is that good sensory localization may be possible even though preinjury patterns of cortical organization are not reestablished.

A third hypothesis is that tactile localization may be recovered through the improved use of whatever limited, but normal, cortical features are restored after regeneration. The monkeys in the present study had patchlike representations which occupied roughly normal cortical locations and which contained some degree of local topographical organization. Although recovery of representations at correct cortical locations may be 
accidental and limited, their reestablishment could be important. Consistent with this hypothesis, recent studies in owl monkeys suggest that normal cortical organization is reestablished following median nerve crush and regeneration (Wall et al., 1983; Wall et al., 1984). In humans, regeneration after nerve crush commonly results in recovery of normal sensory localization. This suggests that there is a correspondence between recstablishment of normal organizational features and recovery of normal sensory features. The implication of these findings for the present study is that, even if cortical disorder is widespread, sensory reeducation may correct localization errors by effectively improving the use of smaller than normal cortical systems that are accurately reconnected to the skin.

Future tests of these and other hypotheses can improve understanding of the effects of sensory therapy on central recovery by determining whether the sequence of reorganizational changes seen in the present study is followed by a further stage of reeducation-related alterations.

\section{References}

Boeke, J. (1940) Problems of Nervous Anatomy, pp. 28-37, Oxford, London.

Boring, E. G. (1916) Cutaneous sensation after nerve division. Q. J. Exp. Physiol. 10: 1-95.

Brown, A. G., and A. Iggo (1963) The structure and function of cutaneous "touch corpuscles" after nerve crush. J. Physiol. (Lond.) 165: 28-29.

Buchthal, F., and V. Kuhl (1979) Nerve conduction, tactile sensibility, and the electromyogram after suture or compression of peripheral nerve: A longitudinal study in man. J. Neurol. Neurosurg. Psychiatry 42: 436-451.

Burgess, P. R., and K. W. Horch (1973) Specific regeneration of cutaneous fibers in the cat. J. Neurophysiol. 36: 101-114.

Burgess, P. R., K. B. English, K. W. Horch, and L. J. Stensaas (1974) Patterning in the regeneration of Type I cutaneous receptors. J. Physiol. (Lond.) 236: 57-82.

Cavanaugh, M. W. (1951) Quantitative effects of the peripheral innervation area on nerves and spinal ganglion cells. J. Comp. Neurol. 94: 181-219.

Cobb, S. (1919) Cutaneous sensibility in cases of peripheral nerve injury: Epicritic and protopathic hypothesis of Head untenable. Arch. Neurol. Psychiatry 2: 505-517.

Cragg, B. G., and P. K. Thomas (1961) Changes in conduction velocity and fiber size proximal to peripheral nerve lesions. J. Physiol. (Lond.) 157: 315-327.

Darian-Smith, I. (1984) The sense of touch: Performance and peripheral neural processes. In The Handbook of Physiology, Vol. III, J. M. Brookhart and V. B. Mountcastle, eds., Sec. 1, pp. 739-788, American Physiological Society, Bethesda, MD.

Dellon, A. L. (1981) Evaluation of Sensibility and Re-Education of Sensation in the Hand, Williams \& Wilkins, London.

Dellon, A. L., F. G. Witebsky, and R. E. Terrill (1975) The denervated meissner corpuscle. Plast. Reconstruct. Surg. 56: 182-193.

Dykes, R. W., and J. K. Terzis (1979) Reinnervation of glabrous skin in baboons: Properties of cutaneous mechanoreceptors subsequent to nerve crush. J. Neurophysiol. 42: 1461-1478.

English, K. B. (1977) The ultrastructure of cutaneous Type I mechanoreceptors (Haarscheiben) in cats following denervation. J. Comp. Neurol. 172: 137-164.

Flynn, J. E., and W. F. Flynn (1962) Median and ulnar nerve injuries: A long range study with evaluation of the ninhydrin test, sensory and motor returns. Ann. Surg. 156: 1002-1009.

Ford, F. R., and B. Woodhall (1938) Phenomena due to misdirection of regenerating fibers of cranial, spinal, and autonomic nerves. Arch. Surg. 36: $480-496$.

Franz, S. I. (1909) Sensations following nerve division. J. Comp. Neurol. 19: 107-123.

Glees, P., A. Mohiuddin, and A. G. Smith (1949) Transplantation of pacinian bodies in the brain and thigh of the cat. Acta Anatom. 7: 213-224.

Hallin, R. G., Z. Wiesenfeld, and U. Lindblom (1981) Neurophysiological studies on patients with sutured median nerves: Faulty sen- sory localization after nerve regeneration and its physiological correlates. Exp. Neurol. 73: 90-106.

Hawkins, G. L. (1948) Faulty sensory localization in nerve regeneration. J. Neurosurg. 5: 11-18.

Head, H. (1920) Studies in Neurology. Vol. 1. Oxford, London.

Horch, K. W. (1976) Ascending collaterals of cutaneous neurons in the fasciculus gracilis of the cat during peripheral nerve regeneration. Brain Res. 117: 19-32.

Horch, K. W. (1979) Guidance of regrowing sensory axons after cutaneous nerve lesions in the cat. J. Neurophysiol. 42: 1437-1449.

Horch, K. W., and P. R. Burgess (1980) Functional specificity and somatotopic organization during peripheral nerve regeneration. In Nerve Repair and Regeneration, D. L. Jewett and H. R. McCarroll, eds., pp. 105-109, Mosby, St. Louis, MO.

Horch, K. W., and S. J. W. Lisney (1981) On the number and nature of regenerating myelinated axons after lesions of cutaneous nerves in the cat. J. Physiol. (Lond.) 313: 275-286.

Jabaley, M. E., J. E. Burns, B. S. Orcutt, and W. M. Bryant (1976) Comparison of histologic and functional recovery after peripheral nerve repair. J. Hand Surg. 1: 119-130.

Jenkins, W. A., M. Merzenich, J. M. Zook, B. C. Fowler, and M. P. Stryker (1982) The area $3 \mathrm{~b}$ representation of the hand in owl monkeys reorganizes after induction of restricted cortical lesions. Soc. Neurosci. Abstr. 8: 141.

Jenkins, W. M., M. M. Merzenich, and M. T. Ochs (1984) Behaviorally controlled differential use of restricted hand surfaces induce changes in the cortical representation of the hand in area $3 \mathrm{~b}$ of adult owl monkeys. Soc. Neurosci. Abstr. 10:665.

Kaas, J. H., M. M. Merzenich, and H. P. Killackey (1983) The reorganization of somatosensory cortex following peripheral nerve damage in adult and developing mammals. Annu. Rev. Neurosci. 6: 325-356.

Karpati, G., S. Carpenter, and L. Charron (1981) Experimental reinnervation attempts of skeletal muscle cells by non-motor nerves. In Posttraumatic Peripheral Nerve Regeneration, A. Gorio, H. Millesi, and S. Mingrino, eds., pp. 495-506, Raven, New York.

Langley, J. N. (1908) Effect on nail growth and sensation of section of a cutaneous digital nerve, and effect on sensation of cocainising a cutaneous nerve of the foot. J. Physiol. (Lond.) 36: xlv-xlvi.

Lee, F. C. (1936) A study of the pacinian corpuscle. J. Comp. Neurol. 64: $497-522$.

Lisney, S. J. W. (1983a) Changes in the somatotopic organization of the cat lumbar spinal cord following peripheral nerve transection and regeneration. Brain Res. 259: 31-39.

Lisney, S. J. W. (1983b) The cat lumbar spinal cord somatotopic map is unchanged after peripheral nerve crush and regeneration. Brain Res. 271: 166-169.

Mackel, R., E. Kunesch, F. Waldhor, and A. Struppler (1983a) Reinnervation of mechanoreceptors in the human glabrous skin following peripheral nerve repair. Brain Res. 268:49-65.

Mackel, R., E. E. Brink, and G. Wittkowsky (1983b) Transient properties of mechanoreceptive afferents during reinnervation of the glabrous skin of the human. Soc. Neurosci. Abstr. 9: 51.

Merzenich, M. M., J. H. Kaas, M. Sur, and C. S. Lin (1978) Double representation of the body surface within cytoarchitectonic Areas $3 \mathrm{~b}$ and 1 in "S-I" in the owl monkey (Aotus trivirgatus). J. Comp. Neurol. 181: 41-74.

Merzenich, M. M., J. H. Kaas, J. T. Wall, R. J. Nelson, M. Sur, and D. J. Felleman (1983a) Topographic reorganization of somatosensory cortical Areas $3 \mathrm{~b}$ and 1 in adult monkeys following restricted deafferentiation. Neuroscience 8: 33-55.

Merzenich, M. M., J. H. Kaas, J. T. Wall, M. Sur, R. J. Nelson, and D. J. Felleman (1983b) Progression of change following median nerve section in the cortical representation of the hand in Areas $3 \mathrm{~b}$ and in 1 adult owl and squirrel monkeys. Neuroscience 10:639-665.

Merzenich, M. M., R. J. Nelson, M. P. Stryker, M. S. Cynader, A. Schoppmann, and J. M. Zook (1984) Somatosensory cortical map changes following digit amputation in adult monkeys. J. Comp. Neurol. 224: 591-605.

Miller, S. H., and I. Rusenas (1976) Changes in primate pacinian corpuscles following volar pad excision and skin grafting. Plast. Reconstruct. Surg. 57: 627-636.

Millesi, H. (1980) Interfascicular nerve repair and secondary repair with nerve grafts. In Nerve Repair and Regeneration. D. L. Jewett and H. R. McCarroll, eds., pp. 299-319, Mosby, St. Louis, MO. 
Mitchell, J. K. (1895) Remote Consequences of Injuries of Nerves and Their Treatment. Examination of the Present Condition of Wounds Received 1863-5 with Illustrative Cases, Lea Brothers, Philadelphia.

Moberg, E. (1962) Criticism and study of methods for examining sensibility in the hand. Neurology 12: 8-19.

Omer, G. E. (1974) Injuries to nerves of the upper extremity. J. Bone Joint Surg. 56A: 1615-1624.

Onne, L. (1962) Recovery of sensibility and sudomotor activity in the hand after nerve suture. Acta Chir. Scand. (Suppl.) 300: 1-69.

Paul, R. L., H. Goodman, and M. Merzenich (1972) Alterations in mechano-receptor input to Brodmann's Areas 1 and 3 of the postcentral hand area of Macaca mulatta after nerve section and regeneration. Brain Res. 39: 1-19.

Poppen, N. K. (1980) Clinical evaluation of the von Frey and two point discrimination tests and correlation with a dynamic test of sensibility. In Nerve Repair and Regeneration, D. L. Jewett and $\mathrm{H}$. R. McCarroll, eds., pp. 252-262, Mosby, St. Louis, MO.

Ranson, S. W. (1906) Retrograde degeneration in the spinal nerves. J. Comp. Neurol. 16: 265-293.

Rosenfalck, A. (1980) Action potentials from sensory nerves in man in nerve damage and during recovery. In Nerve Repair and Regeneration, D. L. Jewett and H. R. McCarroll, eds., pp. 212-219, Mosby, St. Louis, MO.

Schiff, J., and W. R. Loewenstein (1972) Development of a receptor on a foreign nerve fiber in a pacinian corpuscle. Science 177: 712715 .

Seddon, H. J. (1943) Three types of nerve injury. Brain 66: 238-288.

Sharpey-Schafer, E. (1928) The effects of denervation of a cutaneous area. Q. J. Exp. Physiol. 19: 85-107.

Sharpey-Schafer, E. (1930) The permanent results of denervation of a cutaneous area. Q. J. Exp. Physiol. 20: 95-99.

Sperry, R. W. (1945) The problem of central nervous reorganization after nerve regeneration and muscle transposition. Q. Rev. Biol. 20: 311-369.

Stopford, J. S. B. (1926) An explanation of the two-stage recovery of sensation during regeneration of a peripheral nerve. Brain 49: 372386 .

Stopford, J. S. B. (1927) Disturbances of sensation following section and suture of a peripheral nerve. Brain 50: 391-398.

Stromberg, W. B., R. W. McFarlane, J. L. Bell, S. L. Koch, and M. L. Mason (1961) Injury of the median and ulnar nerves. J. Bone Joint Surg. 43A: 717-730.

Sunderland, S. (1978) Nerves and Nerve Injuries, Churchill Livingstone, New York.
Terzis, J. K. (1981) Patterns of cutaneous innervation and reinnervation following nerve transection. In Posttraumatic Peripheral Nerve Regeneration, A. Gorio, H. Millesi, and S. Mingrino, eds., pp. 591610, Raven, New York.

Terzis, J. K., and R. W. Dykes (1980) Reinnervation of glabrous skin in baboons: Properties of cutaneous mechanoreceptors subsequent to nerve transection. J. Neurophysiol. 44: 1214-1225.

Trotter, W., and H. M. Davies (1909) Experimental studies in the innervation of the skin. J. Physiol. (Lond.) 38: 135-247.

Tupper, J. W. (1980) Fascicular nerve repair. In Management of Peripheral Nerve Problems, G. E. Omer and M. Spinner, eds., pp. 380387, W. B. Saunders, Philadelphia, PA.

Waite, P. M. E., and B. G. Cragg (1982) The peripheral and central changes resulting from cutting or crushing the afferent nerve supply to the whiskers. Proc. R. Soc. Lond. [Biol.] 214: 191-211.

Wall, J. T., and J. H. Kaas (1985) Cortical reorganization and sensory recovery following nerve damage and regeneration. In Synaptic Plasticity, C. Cotman, ed., pp. 231-260, Guilford, New York.

Wall, J. T., D. J. Felleman, and J. H. Kaas (1983) Recovery of normal topography in the somatosensory cortex of monkeys after nerve crush and regeneration. Science 221: 771-773.

Wall, J. T., J. H. Kaas, and D. J. Felleman (1985) Functional recovery in the somatosensory cortex of monkeys with regenerated nerves: Replication versus reintegration. In Development, Organization, and Processing in Somatosensory Pathways, M. J. Rowe and W. D. Willis, eds., Alan R. Liss, New York.

Wall, P. D., and M. Devor (1978) Physiology of sensation after peripheral nerve injury, regeneration, and neuroma formation. In Physiology and Pathobiology of Axons, S. G. Waxman, ed., pp. 377-388, Raven, New York.

White, P. F., W. L. Way, and A. J. Trevor (1982) Ketamine-its pharmacology and therapeutic uses. Anesthesiology 56: 119-136.

Wong, W. C., and R. Kanagasuntheram (1971) Early and late effects of median nerve injury on Meissner's and Pacinian corpuscles of the hand of the macaque ( $M$. fascicularis). J. Anat. 109: 135-142.

Wynn-Parry, C. B. (1973) Rehabilitation of the Hand, Butterworths, London.

Zalewski, A. A. (1970) Effects of reinnervation on denervated skeletal muscle by axons of motor, sensory, and sympathetic neurons. Am. J. Physiol. 219: 1675-1679.

Zelena, J. (1981) The fate of Pacinian corpuscles after denervation and reinnervation. In Posttraumatic Peripheral Nerve Regeneration A. Gorio, H. Millesi, and S. Mingrino, eds., pp. 563-571, Raven, New York. 